\title{
ROBUST NUMERICAL APPROXIMATION OF COUPLED STOKES' AND DARCY'S FLOWS APPLIED TO VASCULAR HEMODYNAMICS AND BIOCHEMICAL TRANSPORT*
}

\author{
Carlo D'Angelo ${ }^{1}$ And Paolo Zunino ${ }^{1}$
}

\begin{abstract}
The fully coupled description of blood flow and mass transport in blood vessels requires extremely robust numerical methods. In order to handle the heterogeneous coupling between blood flow and plasma filtration, addressed by means of Navier-Stokes and Darcy's equations, we need to develop a numerical scheme capable to deal with extremely variable parameters, such as the blood viscosity and Darcy's permeability of the arterial walls. In this paper, we describe a finite element method for the approximation of incompressible flow coupled problems. We exploit stabilized mixed finite elements together with Nitsche's type matching conditions that automatically adapt to the coupling of different combinations of coefficients. We study in details the stability of the method using weighted norms, emphasizing the robustness of the stability estimate with respect to the coefficients. We also consider an iterative method to split the coupled heterogeneous problem in possibly homogeneous local problems, and we investigate the spectral properties of suitable preconditioners for the solution of the global as well as local problems. Finally, we present the simulation of the fully coupled blood flow and plasma filtration problems on a realistic geometry of a cardiovascular artery after the implantation of a drug eluting stent (DES). A similar finite element method for mass transport is then employed to study the evolution of the drug released by the DES in the blood stream and in the arterial walls, and the role of plasma filtration on the drug deposition is investigated.
\end{abstract}

Mathematics Subject Classification. 65M60, 76D05, 76Z05, 92C50.

Received November 19, 2009. Revised May 10, 2010.

Published online October 11, 2010.

\section{INTRODUCTION}

Mass transport driven by intramural plasma filtration is the basic process to supply nourishment to tissues of medium/small size arteries. In the last decades, this process has also been exploited to deliver drugs to arteries affected by atherosclerotic pathologies, such as coronary arteries. Drug eluting stents represent a recent and widespread example of this procedure. The design of these devices requires first of all to determine the correct

\footnotetext{
Keywords and phrases. Coupled Stokes/Darcy's problem, biological flows and mass transfer, cardiovascular applications, finite element approximation, interior penalty method, iterative splitting strategy, optimal preconditioning.

* This work has been supported by the Italian Institute of Technology with the project NanoBiotechnology - Models and Methods for Degradable Materials, and by the ERC Advanced Grant No. 227058 MATHCARD - Mathematical Modelling and Simulation of the Cardiovascular System.

1 MOX - Modellistica e Calcolo Scientifico, Dipartimento di Matematica "F. Brioschi", Politecnico di Milano, Piazza Leonardo da Vinci 32, 20133 Milano, Italy. carlo.dangelo@polimi.it; paolo.zunino@polimi.it
} 
drug dosage and release rate, which mainly depend on the transport properties of the substrate releasing the drug as well as on the biochemical interaction between the drug and the arterial tissue. More recently, preliminary computational studies $[2,25]$ have put into evidence that blood flow local features and drug deposition into the artery are correlated. These phenomena, may also significantly interact with intramural plasma filtration. However, to our knowledge, such interplay has not yet been deeply investigated.

In this work, we discuss a finite element scheme using interior penalty techniques to solve coupled problems in a domain accounting for both the arterial lumen and the arterial wall. Specifically, we present a finite element method for the coupling of blood flow and intramural plasma filtration in rigid arteries. Under the assumption of Newtonian rheology, the former is described by the Navier-Stokes equations, while the latter is modeled by the Darcy's law. Suitable interface conditions are then set at the interface between different subregions.

The development of a finite element scheme tailored to handle the heterogeneous nature of the global problem is not trivial. To address heterogeneous coefficients, we have considered in [14] a multi-domain formulation, in which the global domain $\Omega$ is divided in $N$ subregions $\Omega_{i}, i=1, \ldots, N$, characterized by hydraulic resistances $\eta_{i}$ and dynamic viscosities $\nu_{i}$. A unified continuous-discontinuous finite element method for the numerical approximation of the coupled incompressible flow problem was introduced, exploiting a weighted interior penalty formulation, together with an iterative splitting (block-Jacobi) method for the solution of the discrete problem based on $N$ local solvers. The challenge of such unified general formulation is related to the coupling between heterogeneous problems, as it happens, for instance, when viscosity is very small or possibly vanishing on one of the subdomains. In this regard, the analysis of the method introduced in [14] encompasses the whole range of admissible parameters $\eta_{i}$ and $\nu_{i}$; moreover, the convergence of the proposed iterative method was proved.

In this paper, more precisely in Section 2, we consider a similar method, introducing suitable weights in the variational formulation that make the method robust with respect to the coefficients of the problem. The robustness of the stability estimate is investigated in details using suitable weighted norms. In Section 3 we propose preconditioning strategies for the local or global problems, we study their spectral properties and we assess their performance, showing that they are effective tools to be used in the framework of the iterative splitting method. We extend the classical preconditioners based for the Schur complement to our stabilized scheme (along the lines presented in [11]; see also [24,37]), and assess the performance of the counterparts of pressure matrix, Cahouet-Chabard as well as monolithic block-diagonal preconditioners applied to the heterogeneous problems under consideration.

The final aim of this work is to couple blood flow and mass transfer. For this reason, in Section 4 we adapt a continuous-discontinuous stabilized finite element scheme, proposed in [8], to the approximation of the coupled advection-diffusion equations governing the drug transport in the lumen and in the arterial wall.

Finally, in Section 5 we perform a computational analysis of drug release from DES. The study of drug eluting stents is challenging from both the modelling and the computational points of view. On one side, a thorough derivation of mathematical models describing drug release from stents has been recently addressed in $[17,21,32,38,42]$. On the other side, several efforts have been devoted to achieve a computational analysis of the problem. Concerning the mechanical analysis of stent deformation, we refer to [30,31]. For the analysis of how stents perturb blood flow and wall shear stresses we mention, among others [3]. Another relevant topic in this context is the development of reduced models for mass transfer. We refer to [15] for an example applied to DES, based on the ideas introduced in [12].

The coupled roles of stent expansion, blood flow and drug release have been only recently and partially investigated. We refer to $[2,25]$ for a bio-medical overview of the problem, supported by computational results, and to [44] for a fully three-dimensional computational analysis. In the latter work, however, the plasma filtration was not considered. For this reason, we concentrate our efforts on the coupling of the luminal flow inside a blood vessel with plasma filtration in the arterial wall, in a complex geometry characterized by an endovascular stent implant interfering with the flow, and show that our method is able to effectively deal with this heterogeneous problem. 


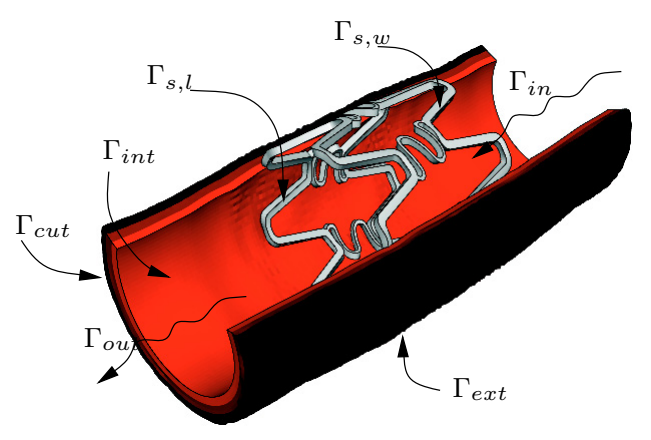

FiguRE 1. The lumen and the arterial wall with boundaries.

We conclude observing that the application of the proposed numerical approximation scheme is not restricted to the analysis of DES. In the framework of cardiovascular applications, the model and its discretization technique find, for instance, a natural application in the study of atherosclerosis. More generally, our scheme could be exploited in very different contexts, such as the simulation of free flows coupled with groundwater filtration in geophysics.

\section{Modelling ANd Approximation of COUPled Blood Flow}

Let us consider the physical domains represented in Figure 1, describing a stented coronary artery. We denote by $\Omega_{l}$ the lumen, that is the domain occupied by blood, and by $\Omega_{w}$ the arterial wall. Let $\partial \Omega_{l}=$ $\Gamma_{\text {in }} \cup \Gamma_{\text {out }} \cup \Gamma_{\text {int }} \cup \Gamma_{s, l}$, where $\Gamma_{\text {in }}$ is the inflow of the lumen, $\Gamma_{\text {out }}$ is the outflow, $\Gamma_{\text {int }}$ is the interface between the lumen and the wall, and $\Gamma_{s, l}$ is the interface between the lumen and the stent.

Analogously, let $\partial \Omega_{w}=\Gamma_{\text {int }} \cup \Gamma_{\text {ext }} \cup \Gamma_{\text {cut }} \cup \Gamma_{s, w}$, where $\Gamma_{\text {ext }}$ is the exterior part of the vessel, $\Gamma_{\text {cut }}$ is the artificial wall boundary, and $\Gamma_{s, w}$ is the interface between the wall and the stent. The exterior unit normal vectors on $\partial \Omega_{l}, \partial \Omega_{w}$, will be denoted respectively by $\mathbf{n}_{l}, \mathbf{n}_{w}$.

\subsection{Blood flow models}

Let us denote by $\mathbf{u}_{i}$ the blood velocity and by $p_{i}$ the blood pressure (normalized with respect to the fluid density) in the arterial lumen $(i=l)$ and in the arterial wall $(i=w)$. In the most general case, we will assume the unsteady Navier-Stokes equations for blood flow in the lumen and the Darcy's model for the filtration of plasma through the wall,

$$
\begin{aligned}
\frac{\partial \mathbf{u}_{l}}{\partial t}-\nu \Delta \mathbf{u}_{l}+\left(\mathbf{u}_{l} \cdot \nabla\right) \mathbf{u}_{l}+\nabla p_{l}=\mathbf{0} & \text { in } \Omega_{l} \times(0, T), \\
\nabla \cdot \mathbf{u}_{l}=0 & \text { in } \Omega_{l} \times(0, T), \\
\eta \mathbf{u}_{w}+\nabla p_{w}=\mathbf{0} & \text { in } \Omega_{w} \times(0, T), \\
\nabla \cdot \mathbf{u}_{w}=0 & \text { in } \Omega_{w} \times(0, T) .
\end{aligned}
$$

Models (2.1), (2.2) and in particular the validity of Darcy's equation for plasma filtration in the arterial wall, is acceptable under the simplificative assumption that the complex multilayer structure of the coronary arteries can be approximated as an isotropic homogeneous medium. We refer to [33] for a review of possible generalization of the filtration model. For the forthcoming numerical simulations, we decide to simply refer to steady NavierStokes equations. We observe that coronary arteries, where DES are typically implanted, can be classified as small and medium sized arteries. In this case, the characteristic Reynolds number is low, so that flow is stable and laminar (see [20] and references therein). Furthermore, to analyze the drug release process on a significant 
time scale, we need to consider a time period containing several thousands of heartbeats, because in general DES are designed to release the drug very slowly. By consequence, the problem at hand features multiple scales with respect to time. Although a detailed analysis of stability and convergence of standard finite difference time advancing schemes applied to problem (2.1) is in preparation [16], the numerical approximation of coupled blood flow and drug release would be inefficient and involve a very high computational effort, because a time step suitable for the small time scale must be applied to achieve a long term simulation. A possibility to overcome this limitation may be represented by the so-called projective integration schemes, see for instance [27] and references therein. However, the development of a specific version of such schemes that would be suitable to our case goes beyond the scope of this work.

In equations (2.1) and (2.2), $\nu$ and $\eta$ are respectively the fluid dynamic viscosity and the hydraulic resistance of the arterial wall. Regarding the boundary conditions, we assign the blood velocity $\mathbf{U}_{l, \text { in }}$ at the inflow boundary $\Gamma_{\text {in }}$, we prescribe zero external stress at the outflow $\Gamma_{\text {out }}$ and a given external pressure $p_{\text {ext }}$ on the exterior part of the wall $\Gamma_{\text {ext }}$, and impose vanishing normal velocity on the artificial boundaries. As interface conditions on $\Gamma_{\text {int }}$, we assume the continuity of the normal component of the velocity and the continuity of the normal component of the normal stress. We also require the tangential component of the lumenal velocity to be zero. Alternatively, a Beavers-Joseph-Saffman law [34] for the jump of the tangential components of the normal stress may be assumed. However, we prefer to consider the limit case of very high frictional stresses, i.e. no slip conditions, because the friction coefficient is often only poorly estimated. The previous assumptions correspond to the following boundary and coupling conditions,

$$
\begin{array}{rlrl}
\mathbf{u}_{l}-\mathbf{U}_{l, \text { in }}=\mathbf{0} & \text { on } \Gamma_{\text {in }}, & \sigma_{w} \mathbf{n}_{w}-p_{\text {ext }} \mathbf{n}_{w}=\mathbf{0} & \text { on } \Gamma_{\text {ext }} \\
\mathbf{u}_{l}=\mathbf{0} & \text { on } \Gamma_{s, l}, & \mathbf{u}_{w} \cdot \mathbf{n}_{w}=0 & \text { on } \Gamma_{s, w} \\
\sigma_{l} \mathbf{n}_{l}=\mathbf{0} & \text { on } \Gamma_{\text {out }}, & \mathbf{u}_{w} \cdot \mathbf{n}_{w}=0 & \text { on } \Gamma_{\text {cut }} \\
& \mathbf{n}_{l}^{T} \sigma_{l} \mathbf{n}_{l}-\mathbf{n}_{w}^{T} \sigma_{w} \mathbf{n}_{w}=0 & \text { on } \Gamma_{\text {int }}, \\
\mathbf{u}_{l} \cdot \mathbf{n}_{l}+\mathbf{u}_{w} \cdot \mathbf{n}_{w}=0 & \text { on } \Gamma_{\text {int }}, \\
\mathbf{n}_{w} \times \mathbf{u}_{l}=\mathbf{0} & \text { on } \Gamma_{\text {int }},
\end{array}
$$

where $\sigma_{l}=p_{l} \mathrm{I}-\nu \nabla \mathbf{u}_{l}, \sigma_{w}=p_{w} \mathrm{I}$ are the Cauchy stress tensors (we denote by I the identity tensor), and $\mathbf{n}_{i}$ is the outward unit normal on $\partial \Omega_{i}, i \in\{l, w\}$.

Problem (2.1) is time-dependent and nonlinear. We consider implicit time advancing schemes (i.e. backward Euler) with time step $\tau$, and Picard's iterations for the treatment of the nonlinear advective term of the NavierStokes equations. Conversely, the Darcy's problem (2.2) depends on time only through interface conditions, and it is linear. If a splitting method is adopted to solve the global nonlinear problem by separately solving the local problems on each subdomain $\Omega_{i}$ at each time step, the Picard's method can be included in the iterations of the splitting scheme by evaluating the advection field at the previous iteration. In this case, the abstract problem that has to be solved at each iteration of the splitting scheme has the following form,

$$
\begin{aligned}
\eta_{i} \mathbf{u}_{i}-\nu_{i} \Delta \mathbf{u}_{i}+\left(\mathbf{w}_{i} \cdot \nabla\right) \mathbf{u}_{i}+\nabla p_{i}=\mathbf{f}_{i} & \text { in } \Omega_{i}, \\
\nabla \cdot \mathbf{u}_{i}=0 & \text { in } \Omega_{i},
\end{aligned}
$$

complemented with boundary and interface conditions (2.3) and (2.4), where $\mathbf{w}_{i}$ are given advection fields for each $i=l, w$. If the backward Euler time advancing scheme is considered, for the luminal flow problem we have $\eta_{l}=\frac{1}{\tau}, \nu_{l}=\nu, \mathbf{f}_{l}=\frac{1}{\tau} \mathbf{u}_{l}^{(k-1)}$, whilst for the arterial wall filtration problem $\nu_{w}=0, \mathbf{w}_{w}=\mathbf{0}, \mathbf{f}_{w}=\mathbf{0}$. In particular, the time discretization of the blood momentum equation gives rise to an additional hydraulic resistance term $\eta_{l}$ (the "inverse" of a permeability) corresponding to the inverse time step. For this reason, we will focus only on the approximation of the steady problem (2.5), since it is clear how to proceed when a time dependent problem is considered. In each subregion we introduce a strictly positive coefficient $\mu_{i}=\nu_{i}+\eta_{i}$, with $i=l, w$. For physiological values of the model parameters, we refer the reader to Section 5 . 
For the analysis of problem (2.5) with non negative viscosity $\nu_{i} \geq 0$, we follow the approach proposed in [1], where a unified analysis of Stokes' and Darcy's problems is addressed. In particular, we look at the Darcy's problem as the singular limit for $\nu_{i} \rightarrow 0$ of (2.5). In this case, the natural spaces for the variational formulation are $\mathbf{V}_{w}=\mathbf{H}_{\text {div }}\left(\Omega_{w}\right), Q_{w}=L^{2}\left(\Omega_{w}\right)$ for the Darcy's problem and $\mathbf{V}_{l}=\left[H^{1}\left(\Omega_{l}\right)\right]^{d}, Q_{l}=L^{2}\left(\Omega_{l}\right)$ for the generalized Stokes/Oseen's problem. In order to treat in a unified way the abrupt transition between the two cases, we introduce the spaces $\mathbf{V}_{i}$ as the completion of $C^{\infty}\left(\Omega_{i}\right)^{d}$ with respect to the Sobolev norm defined by

$$
\|\mathbf{u}\|_{\mathbf{V}_{i}}^{2}=\int_{\Omega_{i}}\left(\eta_{i} \mathbf{u} \cdot \mathbf{u}+\mu_{i}(\nabla \cdot \mathbf{u})^{2}+\nu_{i} \nabla \mathbf{u}: \nabla \mathbf{u}\right) .
$$

We point out that $\mathbf{V}_{i}$ is isomorphic to $\left[H^{1}\left(\Omega_{i}\right)\right]^{d}$ if $\nu_{i}>0$, or to $\mathbf{H}_{\mathrm{div}}\left(\Omega_{i}\right)$ if $\nu_{i}=0$. Then, we look for a weak solution of (2.5) in the spaces $\mathbf{V}_{i} \times Q_{i}$, where $Q_{i}=L^{2}\left(\Omega_{i}\right)$, equipped with the broken norm

$$
\left\|\mathbf{u}_{i}, p_{i}\right\|\|:=\| \mathbf{u}_{i}\left\|_{\mathbf{V}_{i}}^{2}+\right\| \mu_{i}^{-\frac{1}{2}} p_{i} \|_{L^{2}\left(\Omega_{i}\right)}
$$

Theorem 2.1 of [1] shows that problem (2.5) is stable in the norm (2.6).

Concerning $\mathbf{w}_{i}$, we assume that it is divergence free, even though most of the results of this work will still hold true for $\left\|\nabla \cdot \mathbf{w}_{i}\right\|_{0, \Omega_{i}}$ small enough. Moreover, we assume that $\Gamma_{\text {in }}$ is the only inflow boundary, more precisely $\left\{\mathbf{w}_{i} \cdot \mathbf{n}_{i} \leq 0\right\} \subseteq \Gamma_{\text {in }}$. We notice that both assumptions may not be exactly satisfied when $\mathbf{w}_{i}$ is given by a discrete velocity field computed by means of an iterative process to solve the associated nonlinear Navier-Stokes problem (or to solve the coupled lumen-wall problem). However, these minor exceptions do not compromise the validity of our analysis.

\subsection{Finite element discretization with penalties}

The abstract problem (2.5) is an extension (i.e. including an advective term) of the generalized Stokes' problems considered in [14], and can be treated using similar techniques, based on a $H^{1}$-conforming approximation for velocities on each subdomain together with Nitsche's type penalty method for the coupling between different subproblems. Simultaneously, for a more accurate approximation of the divergence free constraint, we will seek the pressure into a fully discontinuous finite element space. The combination of discontinuous velocities at the subdomain interface with fully discontinuous pressures leads to a continuous-discontinuous Galerkin type penalty method.

We assume that each $\Omega_{i}$ is a convex polygonal domain, equipped with a family of quasi-uniform triangulations $\mathcal{T}_{h, i}$ made of affine simplexes $K$ that are conforming on $\Gamma_{\text {int }}$. Let $h$ be their characteristic size. We also denote with $\mathcal{F}_{h, i}$ the set of all interior faces $F$ of $\mathcal{T}_{h, i}$, and set $\mathcal{T}_{h}=\cup_{i} \mathcal{T}_{h, i}, \mathcal{F}_{h}=\cup_{i} \mathcal{F}_{h, i}$.

For the local approximation on each subregion we consider a $\left(\mathbb{P}^{k}\right.$, discontinuous $\left.\mathbb{P}^{k-1}\right)$ finite element pair,

$$
\begin{aligned}
V_{h, i} & :=\left\{v_{h} \in C\left(\Omega_{i}\right):\left.v_{h}\right|_{K} \in \mathbb{P}^{k}(K), \forall K \in \mathcal{T}_{h, i}\right\}, \quad \mathbf{V}_{h, i}=\left[V_{h, i}\right]^{d}, \\
Q_{h, i} & :=\left\{q_{h} \in L^{2}\left(\Omega_{i}\right):\left.q_{h}\right|_{K} \in \mathbb{P}^{k-1}(K), \forall K \in \mathcal{T}_{h, i}\right\}
\end{aligned}
$$

where $k \geq 1$. As global approximation spaces, we consider

$$
\mathbf{V}_{h}:=\bigoplus_{i=1}^{N} \mathbf{V}_{h, i}, \quad Q_{h}:=\bigoplus_{i=1}^{N} Q_{h, i}
$$

Note that the pressure will be uniquely determined by the prescribed external stresses in (2.3).

We define the jump of any finite element function $\phi_{h}$ across any (internal) face $F$ of the computational grid in the usual way,

$$
\llbracket \phi_{h} \rrbracket(\mathbf{x}):=\lim _{\delta \rightarrow 0}\left[\phi_{h}\left(\mathbf{x}-\delta \mathbf{n}_{F}\right)-\phi_{h}\left(\mathbf{x}+\delta \mathbf{n}_{F}\right)\right], \quad \mathbf{x} \in F .
$$


Here, $\phi_{h}$ can be a scalar $Q_{h}$ function or a vector $\mathbf{V}_{h}$ function. The orientation of the normal $\mathbf{n}_{F}$ is arbitrary and does not influence the method. Finally, we denote $h_{F}=\operatorname{diam}(F)$ the diameter of any face $F$. With little abuse of notation, we also denote $h_{F}$ a piecewise constant function defined on $\mathcal{F}_{h}$, taking the value $\operatorname{diam}(F)$ on each face $F$. We also define the weighted and conjugate weighted averages on $\Gamma_{\text {int }}$,

$$
\left\{\phi_{h}\right\}_{w}:=\sum_{i \in\{l, w\}} w_{i} \phi_{h, i}, \quad\left\{\phi_{h}\right\}^{w}:=\sum_{i \in\{l, w\}} \bar{w}_{i} \phi_{h, i}
$$

where $w=\left(w_{l}, w_{w}\right)$ are suitable weights, such that $w_{l}+w_{w}=1$, and $\bar{w}_{i}=1-w_{i}$ are the conjugate weights. We also denote with $\left\{\phi_{h}\right\}$ the standard arithmetic average.

In this work, the choice of the weights is arbitrary and it does not influence the consistency and unique solvability of our finite element scheme. However, as already shown in $[8,14]$, a specific choice of the weights affects the robustness of the scheme with respect to the heterogeneity of the coefficients across the interface. The choice of the weights is also relevant for the performance of the algebraic solvers for the coupled problem, which will be discussed later on. For these reasons, we will consider a specific pair, that will be shown to be particularly effective, given by

$$
w_{l}=\frac{\mu_{w}}{\mu_{l}+\mu_{w}}, \quad w_{w}=\frac{\mu_{l}}{\mu_{l}+\mu_{w}},
$$

for which we have $\left\{\mu \phi_{h}\right\}_{w}=\{\mu\}_{w}\left\{\phi_{h}\right\}$.

Let us denote by $\Gamma_{i}^{D} \subset \partial \Omega_{i}$ the Dirichlet boundaries (see Eq. (2.3)), that are $\Gamma_{l}^{D}=\Gamma_{\text {in }} \cup \Gamma_{s, l}$ and $\Gamma_{w}^{D}=\Gamma_{\text {cut }} \cup$ $\Gamma_{s, w}$, and set $\Gamma^{D}=\Gamma_{l}^{D} \cup \Gamma_{w}^{D}$. For $\mathbf{u}_{h}=\left(\mathbf{u}_{h, l}, \mathbf{u}_{h, w}\right) \in \mathbf{V}_{h}, \mathbf{v}_{h}=\left(\mathbf{v}_{h, l}, \mathbf{v}_{h, w}\right) \in \mathbf{V}_{h}$, and $p_{h}=\left(p_{h, l}, p_{h, w}\right) \in Q_{h}$, $q_{h}=\left(q_{h, l}, q_{h, w}\right) \in Q_{h}$ we define the following forms,

$$
\begin{aligned}
a_{i}^{(b f)}\left(\mathbf{u}_{h, i}, \mathbf{v}_{h, i} ; \mathbf{w}_{i}\right):= & \int_{\Omega_{i}}\left(\eta_{i} \mathbf{u}_{h, l} \cdot \mathbf{v}_{h, i}+\nu_{i} \nabla \mathbf{u}_{h, i}: \nabla \mathbf{v}_{h, i}+\left(\mathbf{w}_{i} \cdot \nabla \mathbf{u}_{h, i}\right) \cdot \mathbf{v}_{h, i}\right) \\
& +\int_{\Gamma_{i}^{D}}\left(\frac{1}{2}\left(\left|\mathbf{w}_{i} \cdot \mathbf{n}_{i}\right|-\mathbf{w}_{i} \cdot \mathbf{n}_{i}\right)+\gamma_{u} h_{F}^{-1} \nu_{i}\right) \mathbf{u}_{h, i} \cdot \mathbf{v}_{h, i} \\
& +\int_{\Gamma_{i}^{D}} \gamma_{u} h_{F}^{-1} \mu_{i}\left(\mathbf{u}_{h, i} \cdot \mathbf{n}_{i}\right)\left(\mathbf{v}_{h, i} \cdot \mathbf{n}_{i}\right) \\
& -\int_{\Gamma_{i}^{D}}\left(\nu_{i} \nabla \mathbf{u}_{h, i} \mathbf{n} \cdot \mathbf{v}_{h, i}+\nu_{i} \nabla \mathbf{v}_{h, i} \mathbf{n} \cdot \mathbf{u}_{h, i}\right) \\
& +\int_{\Gamma_{\mathrm{int}}} \gamma_{u} h_{F}^{-1} \nu_{i}\left(\mathbf{n} \times \mathbf{u}_{h, i}\right) \cdot\left(\mathbf{n} \times \mathbf{v}_{h, i}\right), \\
b_{i}\left(p_{h, i}, \mathbf{v}_{h, i}\right) & :=-\int_{\Omega_{i}} p_{h, i} \nabla \cdot \mathbf{v}_{h, i}+\int_{\Gamma_{i}^{D}} p_{h, i} \mathbf{v}_{h, i} \cdot \mathbf{n}_{i}, \\
c^{(b f)}\left(\mathbf{u}_{h}, \mathbf{v}_{h}\right):= & \int_{\Gamma_{\mathrm{int}}}\left(\gamma_{u} h_{F}^{-1}\{\mu\}_{w} \llbracket \mathbf{u}_{h} \cdot \mathbf{n} \rrbracket \llbracket \mathbf{v}_{h} \cdot \mathbf{n} \rrbracket\right. \\
& \left.-\left\{\nu \mathbf{n}^{T} \nabla \mathbf{u}_{h} \mathbf{n}\right\}_{w} \llbracket \mathbf{v}_{h} \cdot \mathbf{n} \rrbracket-\left\{\nu \mathbf{n}^{T} \nabla \mathbf{v}_{h} \mathbf{n}\right\}_{w} \llbracket \mathbf{u}_{h} \cdot \mathbf{n} \rrbracket\right), \\
d\left(p_{h}, \mathbf{v}_{h}\right):= & \int_{\Gamma_{\mathrm{int}}}\left\{p_{h}\right\}_{w} \llbracket \mathbf{v}_{h} \cdot \mathbf{n} \rrbracket, \\
j_{i}^{(p)}\left(p_{h, i}, q_{h, i}\right):= & \int_{\mathcal{F}_{h, i}} \gamma_{p} h_{F} \mu_{i}^{-1} \llbracket p_{h, i} \rrbracket \llbracket q_{h, i} \rrbracket, \\
j_{i}^{(\mathbf{w})}\left(\mathbf{u}_{h, i}, \mathbf{v}_{h, i} ; \mathbf{w}_{i}\right):= & \int_{\mathcal{F}_{h, i}} \gamma_{\mathbf{w}} h_{F}^{2}\left\|\mathbf{w}_{i} \cdot \mathbf{n}\right\|{ }_{\infty, F} \llbracket \nabla \mathbf{u}_{h, i} \mathbf{n} \rrbracket \cdot \llbracket \nabla \mathbf{v}_{h, i} \mathbf{n} \rrbracket,
\end{aligned}
$$


where $\mathbf{n}$ is a unit normal vector on $\Gamma_{\text {int }}$, defined either as $\mathbf{n}=\mathbf{n}_{l}$ or $\mathbf{n}=\mathbf{n}_{w}$ (all properties of our scheme being independent of this choice), $\gamma_{u}, \gamma_{p}$ and $\gamma_{\mathbf{w}}$ are constant parameters that guarantee the stability of the method. In particular, $\gamma_{\mathbf{w}}$ is associated to an interior penalty stabilization of the convective term, see [9]. Superscripts (bf) ("blood flow") are used to avoid confusion with similar bilinear forms (denoted by (mt)) that we will introduce in Section 4 for the approximation of the mass transfer problem. Note also that forms $c^{(b f)}$ and $d$ contain the interface terms corresponding respectively to $a^{(b f)}$ and $b$. Let us introduce

$$
\begin{aligned}
\mathcal{A}^{(b f)}\left(\mathbf{u}_{h}, \mathbf{v}_{h} ; \mathbf{w}\right) & :=\sum_{i=l, w}\left(a_{i}^{(b f)}+j_{i}^{(\mathbf{w})}\right)\left(\mathbf{u}_{h, i}, \mathbf{v}_{h, i} ; \mathbf{w}_{i}\right)+c^{(b f)}\left(\mathbf{u}_{h}, \mathbf{v}_{h}\right) \\
\mathcal{B}\left(p_{h}, \mathbf{v}_{h}\right) & :=\sum_{i \in\{i, l\}} b_{i}\left(p_{h, i}, \mathbf{v}_{h, i}\right)+d\left(p_{h}, \mathbf{v}_{h}\right) \\
j^{(p)}\left(p_{h}, q_{h}\right) & :=\sum_{i \in\{i, l\}} j_{i}^{(p)}\left(p_{h, i}, q_{h, i}\right)
\end{aligned}
$$

and denote the right hand side by

$$
\begin{aligned}
\mathcal{F}^{(b f)}\left(\mathbf{v}_{h}\right):= & \int_{\Omega_{l}} \mathbf{f}_{l} \cdot \mathbf{v}_{h, l}-\int_{\Gamma_{\mathrm{ext}}} p_{\mathrm{ext}} \mathbf{v}_{h, w} \cdot \mathbf{n}_{w} \\
& +\int_{\Gamma_{\mathrm{in}}}\left[\left(\frac{1}{2}\left(\left|\mathbf{w}_{l} \cdot \mathbf{n}_{l}\right|-\mathbf{w}_{l} \cdot \mathbf{n}_{l}\right)+\gamma_{u} h_{F}^{-1} \nu_{l}\right) \mathbf{U}_{l, \text { in }} \cdot \mathbf{v}_{h, l}+\gamma_{u} h_{F}^{-1} \mu_{l} \mathbf{U}_{l, \text { in }} \cdot \mathbf{n}_{l} \mathbf{v}_{h, l} \cdot \mathbf{n}_{l}\right] .
\end{aligned}
$$

Then, the mixed formulation of the discrete problem reads as follows: find $\left(\mathbf{u}_{h}, p_{h}\right) \in \mathbf{V}_{h} \times Q_{h}$ such that,

$$
\begin{cases}\mathcal{A}^{(b f)}\left(\mathbf{u}_{h}, \mathbf{v}_{h} ; \mathbf{w}\right)+\mathcal{B}\left(p_{h}, \mathbf{v}_{h}\right)=\mathcal{F}^{(b f)}\left(\mathbf{v}_{h}\right), & \forall \mathbf{v}_{h} \in \mathbf{V}_{h}, \\ \mathcal{B}\left(q_{h}, \mathbf{u}_{h}\right)-j^{(p)}\left(p_{h}, q_{h}\right)=0, & \forall q_{h} \in Q_{h} .\end{cases}
$$

In order to analyze the consistency of the scheme, we need to ensure that problem (2.18) makes sense for the exact weak solution of the problem. For this reason, we require the following additional regularity,

$$
\left(\mathbf{u}_{l}, p_{l}\right) \in\left[H^{\frac{3}{2}+\epsilon}\left(\Omega_{l}\right)\right]^{d} \times H^{\frac{1}{2}+\epsilon}\left(\Omega_{l}\right), \text { and } p_{w} \in H^{\frac{1}{2}+\epsilon}\left(\Omega_{w}\right) \text { for any } \epsilon>0 .
$$

Before proceeding, let us comment on the motivation for our specific finite element method. We point out that it allows a unified discretization of problem (2.5), which includes the inviscid flow for $\nu_{i}=0$ as a particular case (see also [1]). The main advantage of this approach is its flexibility; multidomain heterogeneous problems are handled by the same method, as opposite to standard coupling strategies, e.g. [18,26]. Moreover, typical features needed for the accurate solution of the mass transport problem, such as the continuity of the advection field across internal edges, and at least the global mass conservation, are satisfied. We note that the proposed method is not locally conservative due to the pressure stabilization. However, discontinuous pressure is usually associated to better mass conservation properties, as confirmed by the error estimate reported in Lemma 2.4 and also shown by the test cases reported in [6].

\subsection{Fundamental properties of the finite element scheme}

In this section we aim to analyze the stability and the convergence of the discrete scheme described in (2.18), with particular emphasis on the dependence of the the stability properties on coefficients $\eta_{i}, \nu_{i}\left(\right.$ and $\left.\mu_{i}\right)$. Conversely, we disregard the robustness of our scheme with respect to highly convection dominated problems. In other words, defining $R_{e}:=\left\|\mathbf{w} \nu^{-1}\right\|_{\infty}$ the Reynolds number, our analysis may not be robust with respect 
to the limit $R_{e} \rightarrow \infty$. However, this is not a major limitation in the hemodynamic conditions of small-medium sized arteries, which typically feature moderate $R_{e}$.

We observe that introducing the product space $\mathbf{W}_{h}:=\mathbf{V}_{h} \times Q_{h}$, the right hand side $\mathcal{G}\left(\mathbf{v}_{h}, q_{h}\right)=\mathcal{F}^{(b f)}\left(\mathbf{v}_{h}\right)$ and the bilinear form

$$
\mathcal{C}\left(\left(\mathbf{u}_{h}, p_{h}\right),\left(\mathbf{v}_{h}, q_{h}\right)\right):=\mathcal{A}^{(b f)}\left(\mathbf{u}_{h}, \mathbf{v}_{h} ; \mathbf{w}_{l}\right)+\mathcal{B}\left(p_{h}, \mathbf{v}_{h}\right)-\mathcal{B}\left(q_{h}, \mathbf{u}_{h}\right)+j^{(p)}\left(p_{h}, q_{h}\right),
$$

problem (2.18) is equivalent to the following: find $\left(\mathbf{u}_{h}, p_{h}\right) \in \mathbf{W}_{h}$ such that,

$$
\mathcal{C}\left(\left(\mathbf{u}_{h}, p_{h}\right),\left(\mathbf{v}_{h}, q_{h}\right)\right)=\mathcal{G}\left(\mathbf{v}_{h}, q_{h}\right), \quad \forall\left(\mathbf{v}_{h}, q_{h}\right) \in \mathbf{W}_{h}
$$

Then, given $\Sigma \subset \partial \Omega_{i}$, for any $\mathbf{v}_{h} \in \mathbf{V}_{h, i}, q_{h, i} \in Q_{h, i}$ we define

$$
\left\|\mathbf{v}_{h, i}\right\|_{ \pm \frac{1}{2}, h, \Sigma}^{2}:=\int_{\Sigma} h_{F}^{\mp 1} \mathbf{v}_{h}^{2}, \quad\left\|\llbracket q_{h, i} \rrbracket\right\|_{ \pm \frac{1}{2}, h, \mathcal{F}_{h, i}}:=\int_{\mathcal{F}_{h, i}} h_{F}^{\mp 1} \llbracket q_{h, i} \rrbracket^{2}
$$

and we introduce suitable norms in $\mathbf{V}_{h}$ and $\mathbf{W}_{h}$ respectively,

$$
\begin{aligned}
&\left\|\mid \mathbf{v}_{h}\right\| \|^{2}:=\sum_{i=l, w}\left[\left\|\eta_{i}^{\frac{1}{2}} \mathbf{v}_{h, i}\right\|_{0, \Omega_{i}}^{2}+\left\|\nu_{i}^{\frac{1}{2}} \nabla \mathbf{v}_{h, i}\right\|_{0, \Omega_{i}}^{2}+\left\|\nu_{i}^{\frac{1}{2}} \mathbf{v}_{h, i}\right\|_{+\frac{1}{2}, h, \Gamma_{i}^{D}}^{2}+\left\|\nu_{i}^{\frac{1}{2}} \mathbf{n} \times \mathbf{v}_{h, i}\right\|_{+\frac{1}{2}, h, \Gamma_{\mathrm{int}}}^{2}\right], \\
&\|\|\left(\mathbf{v}_{h}, q_{h}\right)\|\|^{2}:=\|\| \mathbf{v}_{h}\|\|^{2}+\sum_{i=l, w}\left[\left\|\mu_{i}^{\frac{1}{2}} \nabla \cdot \mathbf{v}_{h, i}\right\|_{0, \Omega_{i}}^{2}+\left\|\mu_{i}^{\frac{1}{2}} \mathbf{v}_{h, i} \cdot \mathbf{n}\right\|_{+\frac{1}{2}, h, \Gamma_{i}^{D}}^{2}\right. \\
&\left.+\left\|\mu_{i}^{-\frac{1}{2}} q_{h, i}\right\|_{0, \Omega_{i}}^{2}+\left\|\mu_{i}^{-\frac{1}{2}} \llbracket q_{h, i} \rrbracket\right\|_{-\frac{1}{2}, h, \mathcal{F}_{h, i}}^{2}\right]+\left\|\{\mu\}_{w}^{\frac{1}{2}} \llbracket \mathbf{v}_{h} \rrbracket \cdot \mathbf{n}\right\|_{+\frac{1}{2}, h, \Gamma_{\mathrm{int}}}^{2}
\end{aligned}
$$

Notice how the different terms are weighted by coefficients $\eta, \nu$ or $\mu$ in the bilinear forms (2.9)-(2.14) as well as in the norms (2.22)-(2.23). These weights will allow us to get a robust stability estimate, as stated by Theorem 2.3.

In the sequel, we will write $x \lesssim y$ if and only if there exists a constant $C>0$, independent of $x, y, h$ and the coefficients $\eta, \nu$ of the problem, such that $x \leq C y$. We will use the symbol $\gtrsim$ similarly. To assess the robustness of our computational method, we will emphasize the dependence of our estimates on the coefficients.

We will use the following inverse inequalities (see [36]) that hold true for all $K \in \mathcal{T}_{h, i}, v_{h} \in Y_{h}^{k}:=\left\{v_{h} \in\right.$ $\left.L^{2}(\Omega):\left.v_{h}\right|_{K} \in \mathbb{P}^{k}(K), \forall K \in \mathcal{T}_{h}\right\}$, and any face $F \subset \partial K$, provided that the mesh is shape regular,

$$
\left(\frac{h_{F}}{k^{2}}\right)^{\frac{1}{2}}\left\|v_{h}\right\|_{0, F} \lesssim\left\|v_{h}\right\|_{0, K}, \quad\left(\frac{h_{K}}{k^{2}}\right)\left\|\nabla v_{h}\right\|_{0, K} \lesssim\left\|v_{h}\right\|_{0, K}
$$

For the ease of notation, we introduce $\left\|\mathbf{v}_{h}\right\|_{0, \cup \Omega_{i}}^{2}:=\sum_{i=l, w}\left\|\mathbf{v}_{h, i}\right\|_{0, \Omega_{i}}^{2}$. For a similar purpose, we introduce the broken Sobolev space $\mathcal{H}^{s}(\Omega):=\bigoplus_{i=1}^{N} H^{s}\left(\Omega_{i}\right)$ equipped with the broken norm $\|v\|_{s, \cup \Omega_{i}}^{2}:=\sum_{i=l, w}\left\|v_{i}\right\|_{s, \Omega_{i}}^{2}$ and the seminorm $|v|_{s, \cup \Omega_{i}}^{2}:=\sum_{i=l, w}\left|v_{i}\right|_{s, \Omega_{i}}^{2}$. We also set $\mathcal{H}_{\Gamma}^{1}(\Omega):=\left\{v \in \mathcal{H}^{1}(\Omega):\left.v\right|_{\Gamma}=0\right\}$, and $\mathcal{H}_{0}^{1}(\Omega):=\mathcal{H}_{\partial \Omega}^{1}(\Omega)$.

Now, we aim to briefly review the fundamental properties of problem (2.21) in terms of consistency, boundedness, stability and convergence. Some parts of this analysis have already been addressed in [14]. For this reason, we will rigorously state all the results providing a detailed proof only for the topics that have not been developed in [14] or elsewhere. 
Lemma 2.1. Consistency. Let $(\mathbf{u}, p)$ be the weak solution of the coupled problem (2.5), (2.3), (2.4), with the additional regularity stated in (2.19), and let $\left(\mathbf{u}_{h}, p_{h}\right) \in \mathbf{W}_{h}$ be the solution of (2.21). Then, we have,

$$
\mathcal{C}\left(\left(\mathbf{u}-\mathbf{u}_{h}, p-p_{h}\right),\left(\mathbf{v}_{h}, q_{h}\right)\right)=0 \quad \forall\left(\mathbf{v}_{h}, q_{h}\right) \in \mathbf{W}_{h} .
$$

Boundedness. For all $\left(\mathbf{u}_{h}, p_{h}\right),\left(\mathbf{v}_{h}, q_{h}\right) \in \mathbf{W}_{h}$, the bilinear form $\mathcal{C}(\cdot, \cdot)$ satisfies,

$$
\mathcal{C}\left(\left(\mathbf{u}_{h}, p_{h}\right),\left(\mathbf{v}_{h}, q_{h}\right)\right) \lesssim\left(1+\mathrm{R}_{\mathrm{e}}\right)||\left|\mathbf{u}_{h}, p_{h}\right|\left\|\left|\left\|\mathbf{v}_{h}, q_{h} \mid\right\| .\right.\right.
$$

Positivity. Provided that $\gamma_{u}$ is large enough, there exists a positive constant $C_{\mathrm{pos}}$, independent of $h, \nu_{i}, \eta_{i}$, and $\mathbf{w}_{i}$, such that,

$$
\mathcal{A}^{(b f)}\left(\mathbf{v}_{h}, \mathbf{v}_{h} ; \mathbf{w}\right) \geq C_{\mathrm{pos}}\left[\left\|\mathbf{v}_{h}\right\|^{2}+\sum_{i=l, w}\left\|\mu_{i}^{\frac{1}{2}} \mathbf{v}_{h, i} \cdot \mathbf{n}\right\|_{+\frac{1}{2}, h, \Gamma_{i}^{D}}^{2}+\left\|\{\mu\}_{w}^{\frac{1}{2}} \llbracket \mathbf{v}_{h} \rrbracket \cdot \mathbf{n}\right\|_{+\frac{1}{2}, h, \Gamma_{\mathrm{int}}}^{2}\right] \quad \forall\left(\mathbf{v}_{h}, q_{h}\right) \in \mathbf{W}_{h} .
$$

Proof. Consistency is easily verified proceeding as in [14], under the assumption that the weak solution $(\mathbf{u}, p)$ of (2.5), (2.3) and (2.4) is regular enough, using the identity $\llbracket(\sigma(\mathbf{u}, p) \mathbf{n}) \cdot \mathbf{v} \rrbracket=\{\sigma(\mathbf{u}, p) \mathbf{n}\}_{w} \cdot \llbracket \mathbf{v} \rrbracket+\llbracket \sigma(\mathbf{u}, p) \mathbf{n} \rrbracket\{\mathbf{v}\}^{w}$, and the stress interface condition (2.4) to cancel the second term on the right hand side of the previous identity.

Boundedness follows from standard Cauchy-Schwarz and inverse inequalities (2.24), the latter being used to estimate the boundary interface and penalty terms. As an example, let us consider the boundedness of the bilinear forms $b_{i}$. First, thanks to the Cauchy-Schwarz inequality,

$$
\begin{aligned}
b_{i}\left(p_{h, i}, \mathbf{v}_{h, i}\right) & =-\int_{\Omega_{i}} p_{h, i} \nabla \cdot \mathbf{v}_{h, i}+\int_{\Gamma_{i}^{D}} p_{h, i} \mathbf{v}_{h, i} \cdot \mathbf{n}_{i} \\
& \leq\left\|\mu_{i}^{-\frac{1}{2}} p_{h, i}\right\|_{0, \Omega_{i}}\left\|\mu_{i}^{\frac{1}{2}} \nabla \cdot \mathbf{v}_{h, i}\right\|_{0, \Omega_{i}}+\left\|\mu_{i}^{-\frac{1}{2}} p_{h, i}\right\|_{-\frac{1}{2}, h, \Gamma_{i}^{D}}\left\|\mu_{i}^{\frac{1}{2}} \mathbf{v}_{h, i} \cdot \mathbf{n}_{i}\right\|_{+\frac{1}{2}, h, \Gamma_{i}^{D}} .
\end{aligned}
$$

Second, from (2.24) we have $\left\|h_{F}^{\frac{1}{2}} \mu^{-\frac{1}{2}} p_{h}\right\|_{0, F} \lesssim\left\|\mu^{-\frac{1}{2}} p_{h}\right\|_{0, K}$ on each face $F$ of each element $K$. Hence, we can estimate $\left\|\mu_{i}^{-\frac{1}{2}} p_{h, i}\right\|_{-\frac{1}{2}, h, \Gamma_{i}^{D}} \lesssim\left\|\mu^{-\frac{1}{2}} p_{h, i}\right\|_{0, \Omega_{i}}$. As a consequence,

$$
b_{i}\left(p_{h, i}, \mathbf{v}_{h, i}\right) \lesssim\left\||| \mathbf{u}_{h}, p_{h}\right\|\left\|\left|\left\|\mathbf{v}_{h}, q_{h}\right\|\right| .\right.
$$

Analogously, all terms that are independent of the advection field $\mathbf{w}$ are bounded with respect to the triple norm, uniformly with respect to the coefficients (we refer the reader to Lem. 4.2 in [14] for a similar analysis). Finally, the advective term is estimated as follows,

$$
\int_{\Omega_{i}}\left(\mathbf{w}_{i} \cdot \nabla \mathbf{u}_{h, i}\right) \cdot \mathbf{v}_{h, i}=\int_{\Omega_{i}}\left(\left(\mathbf{w}_{i} \nu^{-1} \cdot \nabla\right) \nu^{\frac{1}{2}} \mathbf{u}_{h, i}\right) \cdot \nu^{\frac{1}{2}} \mathbf{v}_{h, i} \lesssim \mathrm{R}_{\mathrm{e}}||\left|\mathbf{u}_{h}\left\||||| \mathbf{v}_{h}\right\|\right|,
$$

where we have used the following Poincaré inequality,

$$
\left\|\nu^{\frac{1}{2}} \mathbf{v}_{h, i}\right\|_{0, \Omega_{i}}^{2} \lesssim\left\|\nu^{\frac{1}{2}} \nabla \mathbf{v}_{h, i}\right\|_{0, \Omega_{i}}^{2}+\left\|\nu^{\frac{1}{2}} \mathbf{v}_{h, i}\right\|_{+\frac{1}{2}, h, \Gamma_{i}^{D}}^{2}
$$

We proceed similarly for the upwind terms $\int_{\Gamma_{\text {in }}}\left(\mathbf{w}_{i} \cdot \mathbf{n}_{i}^{-}\right) \mathbf{u}_{i} \cdot \mathbf{v}_{i}$, where $\mathbf{w}_{i} \cdot \mathbf{n}_{i}^{-}=\frac{\left|\mathbf{w}_{i} \cdot \mathbf{n}_{i}\right|-\mathbf{w}_{i} \cdot \mathbf{n}_{i}}{2}$. As a result, the continuity estimate of $\mathcal{A}^{(b f)}$ depends on the Reynolds number $\mathrm{R}_{\mathrm{e}}=\left\|\mathrm{w} \nu^{-1}\right\|_{\infty}$.

The positivity of $\mathcal{A}^{(b f)}$ is proved following [14] for all terms except the advective one. The latter is treated noticing that $\mathbf{w}_{i}$ is divergence free, which implies

$$
\int_{\Omega_{i}}\left(\mathbf{w}_{i} \cdot \nabla\right) \mathbf{v}_{h, i} \cdot \mathbf{v}_{h, i}=\frac{1}{2} \int_{\partial \Omega_{i}}\left(\mathbf{w}_{i} \cdot \mathbf{n}_{i}\right) \mathbf{v}_{h, i} \cdot \mathbf{v}_{h, i} .
$$


Hence, $\Gamma_{\text {in }}$ being the inflow boundary, we can control the advective term thanks to the upwind term,

$$
\int_{\Gamma_{\text {in }}}\left(\mathbf{w}_{i} \cdot \mathbf{n}_{i}^{-}\right) \mathbf{v}_{h, i} \cdot \mathbf{v}_{h, i}+\int_{\Omega_{i}}\left(\mathbf{w}_{i} \cdot \nabla\right) \mathbf{v}_{h, i} \cdot \mathbf{v}_{h, i}=\frac{1}{2} \int_{\partial \Omega_{i}}\left|\mathbf{w}_{i} \cdot \mathbf{n}_{i}\right| \mathbf{v}_{h, i} \cdot \mathbf{v}_{h, i} \geq 0 .
$$

The inf-sup stability of the scheme (2.18) is not a trivial task, because of the continuous-discontinuous nature of the velocity approximation space $\mathbf{V}_{h}$, which admits jumps across the interface $\Gamma_{\text {int }}$. An effective technique to study the inf-sup stability of the scheme when dealing with the homogeneous Dirichlet conditions is discussed in [14], following the Boland-Nicolaides approach, see [4]. This requires, first, to verify that the infsup condition holds uniformly for a pair of subspaces $\mathbf{V}_{h} \times \bar{Q}_{h}$, where $\bar{Q}_{h}$ is the space of constant functions on each subdomain $\Omega_{i}$ that satisfy $\sum_{i \in\{l, w\}} \int_{\Omega_{i}} \bar{q}_{i}=0$. Second, to locally check the inf-sup stability for the local spaces $\mathbf{V}_{h, i}$ and $\tilde{Q}_{h, i}:=Q_{h, i} \cap L_{0}^{2}\left(\Omega_{i}\right)$, where $L_{0}^{2}\left(\Omega_{i}\right)$ is the subspace of $L^{2}$ functions having zero mean value. These two conditions are then combined to recover the global inf-sup stability condition in $\left(\mathbf{V}_{h} \cap\left[\mathcal{H}_{0}^{1}(\Omega)\right]^{d}\right) \times\left(Q_{h} \cap L_{0}^{2}(\Omega)\right)$.

Let $\Gamma_{i}^{N}=\partial \Omega_{i} \backslash\left(\Gamma^{D} \cup \Gamma_{\text {int }}\right) \neq \emptyset$ be the external boundaries of each domain where the stresses are prescribed (i.e., $\Gamma_{l}^{N}=\Gamma_{\text {out }}, \Gamma_{w}^{N}=\Gamma_{\text {ext }}$ ). In the particular case $\Gamma_{i}^{N} \neq \emptyset$ for $i=l$, w, a similar but simpler procedure can be used to obtain the global inf-sup condition on $\left(\mathbf{V}_{h} \cap\left[H_{\Gamma^{D} \cup \Gamma_{\text {int }}}^{1}(\Omega)\right]^{d}\right) \times Q_{h}$, where now the pressure can have non-zero mean value over $\Omega$ since we have homogeneous Dirichlet conditions for the velocity on a subset $\Gamma^{D}$ strictly contained in the whole boundary $\partial \Omega$, and external stresses are prescribed on the remaining boundaries. We summarize these results in the following lemma.

Lemma 2.2. Provided that $\Gamma_{i}^{N} \neq \emptyset$ for $i=l$, w, for all $p_{h} \in Q_{h}$ there exists $\mathbf{v}_{p, h} \in \mathbf{V}_{h} \cap\left[H_{\Gamma^{D} \cup \Gamma_{\mathrm{int}}}^{1}(\Omega)\right]^{d}$ such that,

$$
\begin{aligned}
\mathcal{B}\left(p_{h}, \mathbf{v}_{p, h}\right) & \gtrsim\left\|\mu^{-\frac{1}{2}} p_{h}\right\|_{0, \Omega}^{2}-C\left\|\mu^{-\frac{1}{2}} \llbracket p_{h} \rrbracket\right\|_{-\frac{1}{2}, h, \mathcal{F}_{h}}^{2}, \\
\left\|\mu^{\frac{1}{2}} \mathbf{v}_{p, h}\right\|_{1, \Omega} & \lesssim\left\|\mu^{-\frac{1}{2}} p_{h}\right\|_{0, \Omega},
\end{aligned}
$$

where $C$ is a positive constant independent of $h, \nu_{i}, \eta_{i}$.

Proof. For the sake of simplicity, we only consider the lowest order case $(k=1)$, referring the reader to [14] for the extension to $k>1$. We will write $p_{h}=\sum_{i=l, w} \tilde{p}_{h, i}+\bar{p}_{h, i}$, where $\tilde{p}_{h, i} \in Q_{h, i} \cap L_{0}^{2}\left(\Omega_{i}\right), \bar{p}_{h, i} \in Q_{h, i}$ is a constant function on $\Omega_{i}$, and both $\tilde{p}_{h, i}$ and $\bar{p}_{h, i}$ are extended to $Q_{h}$ by zero. We observe that, by means of the surjectivity of the divergence operator from $\left[H_{0}^{1}\left(\Omega_{i}\right)\right]^{d}$ to $Q_{h} \cap L_{0}^{2}\left(\Omega_{i}\right)$, and since $\mu_{i}$ is a positive constant, there exists $\tilde{\mathbf{v}}_{p, i} \in\left[H_{0}^{1}\left(\Omega_{i}\right)\right]^{d}$ such that,

$$
\nabla \cdot \tilde{\mathbf{v}}_{p, i}=-\mu_{i}^{-1} \tilde{p}_{h, i}, \quad\left\|\mu_{i}^{\frac{1}{2}} \tilde{\mathbf{v}}_{p, i}\right\|_{1, \Omega_{i}} \lesssim\left\|\mu_{i}^{-\frac{1}{2}} \tilde{p}_{h, i}\right\|_{0, \Omega_{i}} .
$$

Let us introduce the $H_{0}^{1}$-conformal $L^{2}$-projector $\pi_{h, i}^{H_{0}^{1}}:\left[H_{0}^{1}\left(\Omega_{i}\right)\right]^{d} \rightarrow \mathbf{V}_{h, i} \cap\left[H_{0}^{1}\left(\Omega_{i}\right)\right]^{d}$. Thanks to the $H^{1}$-stability of the $L^{2}$-projection on finite element spaces (see [5]) we have

$$
\left\|\pi_{h, i}^{H_{0}^{1}} \tilde{\mathbf{v}}_{p, i}\right\|_{1, \Omega_{i}} \lesssim\left\|\tilde{\mathbf{v}}_{p, i}\right\|_{1, \Omega_{i}}, \quad\left\|\pi_{h, i}^{H_{0}^{1}} \tilde{\mathbf{v}}_{p, i}-\tilde{\mathbf{v}}_{p, i}\right\|_{0, K} \lesssim h_{K}\left|\tilde{\mathbf{v}}_{p, i}\right|_{1, K},
$$

for any triangle $K \in \mathcal{T}_{h}$. Now, define $\tilde{\mathbf{v}}_{p, h, i}=\pi_{h, i}^{H_{0}^{1}} \tilde{\mathbf{v}}_{p, i}$. Since $\tilde{\mathbf{v}}_{p, h, i} \in\left[H_{0}^{1}\left(\Omega_{i}\right)\right]^{d}$ we have $d_{i}\left(\tilde{p}_{h, i}, \tilde{\mathbf{v}}_{p, h, i}\right)=0$. Now, we proceed as in [7], Theorem 2. By means of (2.24), (2.25), (2.26), applying integration by parts and observing that $\left.\nabla \tilde{p}_{h, i}\right|_{K}=0$ we obtain,

$$
\begin{aligned}
b_{i}\left(\tilde{p}_{h, i}, \tilde{\mathbf{v}}_{p, h, i}\right) & =\left\|\mu_{i}^{-\frac{1}{2}} \tilde{p}_{h, i}\right\|_{0, \Omega_{i}}^{2}-\int_{\mathcal{F}_{h, i}} \llbracket \tilde{p}_{h, i} \rrbracket\left\{\tilde{\mathbf{v}}_{p, h, i} \cdot \mathbf{n}_{F}-\tilde{\mathbf{v}}_{p, i} \cdot \mathbf{n}_{F}\right\} \\
& \gtrsim\left\|\mu_{i}^{-\frac{1}{2}} \tilde{p}_{h, i}\right\|_{0, \Omega_{i}}^{2}-\frac{1}{\epsilon}\left\|\mu_{i}^{-\frac{1}{2}} \llbracket \tilde{p}_{h, i} \rrbracket\right\|_{-\frac{1}{2}, h, \Omega_{i}}^{2}-\epsilon \int_{\mathcal{F}_{h, i}} h_{F}^{-1} \mu_{i}\left\{\tilde{\mathbf{v}}_{p, h, i} \cdot \mathbf{n}_{F}-\tilde{\mathbf{v}}_{p, i} \cdot \mathbf{n}_{F}\right\}^{2},
\end{aligned}
$$


where $\{\cdot\}$ denotes the arithmetic average. Using inverse inequalities (2.24) and (2.26) to estimate the term depending on velocities, we have

$$
\int_{\mathcal{F}_{h, i}} h_{E}^{-1} \mu_{i}\left\{\tilde{\mathbf{v}}_{p, h, i} \cdot \mathbf{n}_{F}-\tilde{\mathbf{v}}_{p, i} \cdot \mathbf{n}_{F}\right\}^{2} \lesssim\left\|\mu_{i}^{\frac{1}{2}} \tilde{\mathbf{v}}_{p, i}\right\|_{1, \Omega_{i}}^{2},
$$

and thanks to $(2.25)$,

$$
b_{i}\left(\tilde{p}_{h, i}, \tilde{\mathbf{v}}_{p, h, i}\right) \geq(1-\epsilon)\left\|\mu_{i}^{-\frac{1}{2}} \tilde{p}_{h, i}\right\|_{0, \Omega_{i}}-\frac{1}{\epsilon}\left\|\mu_{i}^{-\frac{1}{2}} \llbracket \tilde{p}_{h, i} \rrbracket\right\|_{-\frac{1}{2}, h, \mathcal{F}_{h, i}}^{2}
$$

Now, let us consider the piecewise constant function $\bar{p}_{h}=\bar{p}_{h, l}+\bar{p}_{h, w}$. For any $\overline{\mathbf{v}}_{p, h}=\overline{\mathbf{v}}_{p, h, l}+\overline{\mathbf{v}}_{p, h, w}$ with $\overline{\mathbf{v}}_{p, h, i} \in$ $\mathbf{V}_{h, i} \cap\left[H_{\Gamma^{D} \cup \Gamma_{\text {int }}}^{1}\left(\Omega_{i}\right)\right]^{d}$, by observing that $\bar{p}_{h, i}$ is constant on $\Omega_{i}, \overline{\mathbf{v}}_{p, h, i}=\mathbf{0}$ on $\Gamma_{i}^{D} \cup \Gamma_{\text {int }}$ and $\llbracket \overline{\mathbf{v}}_{p, h} \rrbracket_{\mid \Gamma_{\text {int }}}=\mathbf{0}$, after integrating by parts we have

$$
\mathcal{B}\left(\bar{p}_{h}, \overline{\mathbf{v}}_{p, h}\right)=-\sum_{i=l, w} \bar{p}_{h, i} \int_{\Gamma_{i}^{N}} \overline{\mathbf{v}}_{p, h, i} \cdot \mathbf{n}_{i} .
$$

For $i=l, w$, let $\mathbf{h}_{i} \in \mathbf{V}_{h, i}$ be the finite element harmonic lifting such that $\mathbf{h}_{i}=\mathbf{0}$ on $\Gamma_{\operatorname{int}} \cup \Gamma_{i}^{D}$ and $\int_{\Gamma_{i}^{N}} \mathbf{h}_{i} \cdot \mathbf{n}_{i}=1$. Functions $\overline{\mathbf{v}}_{p, h, i}=-\mu_{i}^{-1}\left|\Omega_{i}\right| \bar{p}_{h, i} \mathbf{h}_{i}$ satisfy

$$
\left\|\mu_{i}^{\frac{1}{2}} \overline{\mathbf{v}}_{p, h, i}\right\|_{1, \Omega_{i}}^{2}=\mu_{i}^{-1}\left|\Omega_{i}\right|^{2}\left|\bar{p}_{h, i}\right|^{2}\left\|\mathbf{h}_{i}\right\|_{1, \Omega_{i}}=\left\|\mu_{i}^{-\frac{1}{2}} \bar{p}_{h, i}\right\|_{0, \Omega_{i}}^{2}\left|\Omega_{i}\right|\left\|\mathbf{h}_{i}\right\|_{1, \Omega_{i}} \lesssim\left\|\mu_{i}^{-\frac{1}{2}} \bar{p}_{h, i}\right\|_{0, \Omega_{i}}^{2},
$$

so that we have

$$
\mathcal{B}\left(\bar{p}_{h}, \overline{\mathbf{v}}_{p, h}\right)=\left\|\mu^{-\frac{1}{2}} \bar{p}_{h}\right\|_{0, \Omega}^{2}, \quad\left\|\mu^{\frac{1}{2}} \overline{\mathbf{v}}_{p, h}\right\|_{1, \Omega}^{2} \lesssim\left\|\mu^{-\frac{1}{2}} \bar{p}_{h}\right\|_{0, \Omega}^{2} .
$$

Let be $\tilde{\mathbf{v}}_{p, h}:=\sum_{i=l, w} \tilde{\mathbf{v}}_{p, h, i}$ and $\tilde{p}_{h}:=\sum_{i=l, w} \tilde{p}_{h, i}$. To conclude, let us take $\mathbf{v}_{p, h}=\tilde{\mathbf{v}}_{p, h}+\delta \overline{\mathbf{v}}_{p, h}, \delta>0$. We have $\mathcal{B}\left(\bar{p}_{h, i}, \tilde{\mathbf{v}}_{p, h, i}\right)=0$, which gives

$$
\begin{aligned}
\mathcal{B}\left(p_{h}, \mathbf{v}_{p, h}\right) & =\mathcal{B}\left(\tilde{p}_{h}, \tilde{\mathbf{v}}_{p, h}\right)+\delta \mathcal{B}\left(\tilde{p}_{h}, \overline{\mathbf{v}}_{p, h}\right)+\delta \mathcal{B}\left(\bar{p}_{h}, \overline{\mathbf{v}}_{p, h}\right) \\
& =\delta\left\|\mu^{-\frac{1}{2}} \bar{p}_{h}\right\|_{0, \Omega}^{2}+\sum_{i=l, w}\left[(1-\epsilon)\left\|\mu_{i}^{-\frac{1}{2}} \tilde{p}_{h, i}\right\|_{0, \Omega_{i}}^{2}-\frac{1}{\epsilon}\left\|\mu_{i}^{-\frac{1}{2}} \llbracket \tilde{p}_{h, i} \rrbracket\right\|_{-\frac{1}{2}, h, \Omega_{i}}^{2}\right]+\delta \mathcal{B}\left(\tilde{p}_{h}, \overline{\mathbf{v}}_{p, h}\right) .
\end{aligned}
$$

Thanks to the second estimate in (2.28), for any $\epsilon^{\prime}>0$ we have

$$
\mathcal{B}\left(\tilde{p}_{h}, \overline{\mathbf{v}}_{p, h}\right)=\sum_{i=l, w} \int_{\Omega_{i}} \tilde{p}_{h, i} \nabla \cdot \overline{\mathbf{v}}_{p, h, i} \geq-\left\|\mu^{-\frac{1}{2}} \tilde{p}_{h}\right\|_{0, \Omega}\left\|\mu^{\frac{1}{2}} \overline{\mathbf{v}}_{p, h}\right\|_{1, \Omega} \gtrsim-\frac{1}{\epsilon^{\prime}}\left\|\mu^{-\frac{1}{2}} \tilde{p}_{h}\right\|_{0, \Omega}^{2}-\epsilon^{\prime}\left\|\mu^{-\frac{1}{2}} \bar{p}_{h}\right\|_{0, \Omega}^{2},
$$

so that

$$
\begin{aligned}
\mathcal{B}\left(p_{h}, \mathbf{v}_{p, h}\right) & \gtrsim \delta\left(1-\epsilon^{\prime}\right)\left\|\mu^{-\frac{1}{2}} \bar{p}_{h}\right\|_{0, \Omega}^{2}+\sum_{i=l, w}\left[\left(1-\epsilon-\frac{\delta}{\epsilon^{\prime}}\right)\left\|\mu_{i}^{-\frac{1}{2}} \tilde{p}_{h, i}\right\|_{0, \Omega}^{2}-\frac{1}{\epsilon}\left\|\mu_{i}^{-\frac{1}{2}} \llbracket \tilde{p}_{h, i} \rrbracket\right\|_{-\frac{1}{2}, h, \mathcal{F}_{h, i}}^{2}\right] \\
& \gtrsim\left\|\mu^{-\frac{1}{2}} p_{h}\right\|_{0, \Omega}^{2}-C\left\|\mu^{-\frac{1}{2}} \llbracket \tilde{p}_{h} \rrbracket\right\|_{-\frac{1}{2}, h, \mathcal{F}_{h}}^{2},
\end{aligned}
$$

where the inequality is satisfied taking for instance $\epsilon^{\prime}=\frac{1}{2}, \delta=\epsilon=\frac{1}{4}$.

We can now assess the stability of our finite element method. 
Lemma 2.3. Under the assumptions of Lemmas 2.1 and 2.2 for all $\left(\mathbf{u}_{h}, p_{h}\right) \in \mathbf{W}_{h}$, there exists $\left(\mathbf{v}_{h}, q_{h}\right) \in \mathbf{W}_{h}$ such that

$$
\mathcal{C}\left(\left(\mathbf{u}_{h}, p_{h}\right),\left(\mathbf{v}_{h}, q_{h}\right)\right) \gtrsim \frac{1}{\left(1+\mathrm{R}_{\mathrm{e}}\right)^{2}}\left\|\left(\mathbf{u}_{h}, p_{h}\right)\right\|\left|\left\|||\left(\mathbf{v}_{h}, q_{h}\right)\right\|\right| \mid .
$$

Proof. Let $\mathbf{v}_{p, h}$ be the function associated to $p_{h}$ as in Lemma 2.2. Owing to the property $\nabla \cdot \mathbf{V}_{h, i} \subset Q_{h, i}$, we choose

$$
\left(\mathbf{v}_{h}, q_{h}\right)=\left(\mathbf{u}_{h}+\delta_{1} \mathbf{v}_{p, h}, p_{h}+\delta_{2} \mu \nabla \cdot \mathbf{u}_{h}\right), \quad \delta_{1}, \delta_{2}>0 .
$$

First, we prove that $\mathcal{C}\left(\left(\mathbf{u}_{h}, p_{h}\right),\left(\mathbf{v}_{h}, q_{h}\right)\right) \gtrsim\left(1+\mathrm{R}_{\mathrm{e}}\right)^{-2}\left\|\left|\left(\mathbf{u}_{h}, p_{h}\right) \|\right|^{2}\right.$, then we show that $\|\left|\left(\mathbf{v}_{h}, q_{h}\right)\right|\left\|\lesssim||\left|\left(\mathbf{u}_{h}, p_{h}\right)\right|\right\|$. For the first part, we exploit the bilinearity of $\mathcal{C}(\cdot, \cdot)$ to obtain,

$$
\mathcal{C}\left(\left(\mathbf{u}_{h}, p_{h}\right),\left(\mathbf{v}_{h}, q_{h}\right)\right)=\mathcal{C}\left(\left(\mathbf{u}_{h}, p_{h}\right),\left(\mathbf{u}_{h}, p_{h}\right)\right)+\delta_{1} \mathcal{C}\left(\left(\mathbf{u}_{h}, p_{h}\right),\left(\mathbf{v}_{p, h}, 0\right)\right)+\delta_{2} \mathcal{C}\left(\left(\mathbf{u}_{h}, p_{h}\right),\left(0, \mu \nabla \cdot \mathbf{u}_{h}\right)\right) .
$$

Owing to Lemma 2.1 the first term on the right hand side of (2.29) can be estimated as,

$$
\begin{aligned}
\mathcal{C}\left(\left(\mathbf{u}_{h}, p_{h}\right),\left(\mathbf{u}_{h}, p_{h}\right)\right) \geq & C_{\text {pos }}\left[\left.\left\|\mathbf{u}_{h}\right\|\right|^{2}+\sum_{i=l, w}\left\|\mu_{i}^{\frac{1}{2}} \mathbf{u}_{h, i} \cdot \mathbf{n}\right\|_{+\frac{1}{2}, h, \Gamma_{i}^{D}}^{2}+\left\|\{\mu\}_{w}^{\frac{1}{2}} \llbracket \mathbf{u}_{h} \rrbracket \cdot \mathbf{n}\right\|_{+\frac{1}{2}, h, \Gamma_{\mathrm{int}}}^{2}\right] \\
& +\gamma_{p}\left\|\mu^{-\frac{1}{2}} \llbracket p_{h} \rrbracket\right\|_{-\frac{1}{2}, h, \mathcal{F}_{h}}^{2} .
\end{aligned}
$$

Let us consider the second term of (2.29). Since $\mathbf{v}_{p, h}=\mathbf{0}$ on $\Gamma_{i}^{D} \cup \Gamma_{\text {int }}$, we have $\left\|\left|\mathbf{v}_{p, h}\right|\right\| \leq\left\|\mu^{\frac{1}{2}} \mathbf{v}_{p, h}\right\|_{1, \Omega}$. Hence, using Lemma 2.1 (boundedness), and $\left\|\left|\mathbf{v}_{p, h}\|\mid \lesssim\| \mu^{-\frac{1}{2}} p_{h} \|_{0, \Omega}\right.\right.$, for all $\epsilon_{1}>0$ we get

$$
\mathcal{A}^{(b f)}\left(\mathbf{u}_{h}, \mathbf{v}_{p, h} ; \mathbf{w}\right) \lesssim\left(1+\mathrm{R}_{\mathrm{e}}\right)\left|\left\|\mathbf { u } _ { h } \left|\left\|\left|\left\|\mathbf { v } _ { p , h } \left|\left\|\leq\left(1+\mathrm{R}_{\mathrm{e}}\right)^{2} \frac{C_{1}}{\epsilon_{1}}\right\| \mathbf{u}_{h}\left\|\left.\right|^{2}+C_{1} \epsilon_{1}\right\| \mu^{-\frac{1}{2}} p_{h} \|_{0, \Omega}^{2},\right.\right.\right.\right.\right.\right.\right.
$$

so that

$$
\begin{aligned}
\mathcal{C}\left(\left(\mathbf{u}_{h}, p_{h}\right),\left(\mathbf{v}_{p, h}, 0\right)\right) & =\mathcal{A}^{(b f)}\left(\mathbf{u}_{h}, \mathbf{v}_{p, h} ; \mathbf{w}\right)+\mathcal{B}\left(p_{h}, \mathbf{v}_{p, h}\right) \\
& \geq \mathcal{B}\left(p_{h}, \mathbf{v}_{p, h}\right)-C_{1} \epsilon_{1}\left\|\mu^{-\frac{1}{2}} p_{h}\right\|_{0, \Omega}^{2}-\left(1+\mathrm{R}_{\mathrm{e}}\right)^{2} \frac{C_{1}}{\epsilon_{1}}\left\|\mathbf{u}_{h}\right\| \|^{2} .
\end{aligned}
$$

Then, thanks to Lemma 2.2 we have

$$
\mathcal{C}\left(\left(\mathbf{u}_{h}, p_{h}\right),\left(\mathbf{v}_{p, h}, 0\right)\right) \geq\left(1-C_{1} \epsilon_{1}\right)\left\|\mu^{-\frac{1}{2}} p_{h}\right\|_{0, \Omega}^{2}-C\left\|\mu^{-\frac{1}{2}} \llbracket p_{h} \rrbracket\right\|_{-\frac{1}{2}, h, \mathcal{F}_{h}}^{2}-\left(1+\mathrm{R}_{\mathrm{e}}\right)^{2} \frac{C_{1}}{\epsilon_{1}}\left\|\mid \mathbf{u}_{h}\right\| \|^{2} .
$$

Now consider the third term of (2.29). Thanks to (2.24), it is easily found that $j^{(p)}\left(\mu \nabla \cdot \mathbf{u}_{h}, \mu \nabla \cdot \mathbf{u}_{h}\right) \lesssim$ $\gamma_{p}\left\|\mu^{\frac{1}{2}} \nabla \cdot \mathbf{u}_{h}\right\|_{0, \Omega}^{2}, \int_{\Gamma_{\text {int }}}\left\{\mu \nabla \cdot \mathbf{u}_{h}\right\}_{w} \llbracket \mathbf{u}_{h} \cdot \mathbf{n} \rrbracket \lesssim\left\|\mu^{\frac{1}{2}} \nabla \cdot \mathbf{u}_{h}\right\|_{0, \Omega}^{2}+\left\|\{\mu\}_{w}^{\frac{1}{2}} \llbracket \mathbf{u}_{h} \cdot \mathbf{n} \rrbracket\right\|_{+\frac{1}{2}, h, \Gamma_{\text {int }}}^{2}$. Using the Young inequality and the previous estimates, for any $\epsilon_{2}>0$ the third term on the right hand side of (2.29) is expressed as

$$
\begin{aligned}
& \mathcal{C}\left(\left(\mathbf{u}_{h}, p_{h}\right),\left(0, \mu \nabla \cdot \mathbf{u}_{h}\right)\right)=\left\|\mu^{\frac{1}{2}} \nabla \cdot \mathbf{u}_{h}\right\|_{0, \Omega}^{2}+j^{(p)}\left(p_{h}, \mu \nabla \cdot \mathbf{u}_{h}\right)-\int_{\partial \Omega}\left(\mu \nabla \cdot \mathbf{u}_{h}\right)\left(\mathbf{u}_{h} \cdot \mathbf{n}\right)-\int_{\Gamma_{\mathrm{int}}}\left\{\mu \nabla \cdot \mathbf{u}_{h}\right\}_{w} \llbracket \mathbf{u}_{h} \cdot \mathbf{n} \rrbracket \\
& \geq\left(1-C_{2}\left(\gamma_{p}+2\right) \epsilon_{2}\right)\left\|\mu^{\frac{1}{2}} \nabla \cdot \mathbf{u}_{h}\right\|_{0, \Omega}^{2}-\frac{C_{2}}{\epsilon_{2}}\left[\gamma_{p}\left\|\mu^{-\frac{1}{2}} \llbracket p_{h} \rrbracket\right\|_{-\frac{1}{2}, h, \mathcal{F}_{h}}^{2}\right. \\
& \left.+\left\|\mu^{\frac{1}{2}} \mathbf{u}_{h} \cdot \mathbf{n}\right\|_{+\frac{1}{2}, h, \partial \Omega}^{2}+\left\|\{\mu\}_{w}^{\frac{1}{2}} \llbracket \mathbf{u}_{h} \cdot \mathbf{n} \rrbracket\right\|_{+\frac{1}{2}, h, \Gamma_{\mathrm{int}}}^{2}\right] .
\end{aligned}
$$


Collecting the previous results we obtain the following estimate,

$$
\begin{aligned}
& \mathcal{C}\left(\left(\mathbf{u}_{h}, p_{h}\right),\left(\mathbf{v}_{h}, q_{h}\right)\right) \gtrsim\left(C_{\text {pos }}-\left(1+\mathrm{R}_{\mathrm{e}}\right)^{2} C_{1} \frac{\delta_{1}}{\epsilon_{1}}\right)\left\|\left|\mathbf{u}_{h} \|\right|^{2}\right. \\
& +\left(C_{\mathrm{pos}}-C_{2} \frac{\delta_{2}}{\epsilon_{2}}\right)\left[\left\|\mu^{\frac{1}{2}} \mathbf{u}_{h} \cdot \mathbf{n}\right\|_{+\frac{1}{2}, h, \partial \Omega}^{2}+\left\|\{\mu\}_{w}^{\frac{1}{2}} \llbracket \mathbf{u}_{h} \cdot \mathbf{n} \rrbracket\right\|_{+\frac{1}{2}, h, \Gamma_{\mathrm{int}}}^{2}\right]+\delta_{1}\left(1-C_{1} \epsilon_{1}\right)\left\|\mu^{-\frac{1}{2}} p_{h}\right\|_{0, \Omega}^{2} \\
& +\delta_{2}\left(1-C_{2}\left(\gamma_{p}+2\right) \epsilon_{2}\right)\left\|\mu^{\frac{1}{2}} \nabla \cdot \mathbf{u}_{h}\right\|_{0, \Omega}^{2}+\left[\gamma_{p}\left(1-C_{2} \frac{\delta_{2}}{\epsilon_{2}}\right)-\delta_{1} C\right]\left\|\mu^{-\frac{1}{2}} \llbracket p_{h} \rrbracket\right\|_{-\frac{1}{2}, h, \mathcal{F}_{h}}^{2} .
\end{aligned}
$$

Now, let us choose sufficiently small parameters $\epsilon_{i}$ and $\delta_{i}$ such that all the norms in (2.31) are multiplied by positive coefficients. We have that $\epsilon_{1} \lesssim 1 / C_{1}$ and $\epsilon_{2} \lesssim 1 /\left(C_{2}\left(\gamma_{p}+2\right)\right)$ regardless of the coefficients $\nu, \eta$. Conversely, the constants $\delta_{i}$ depend on $\mathrm{R}_{\mathrm{e}}$ : we have to chose $\delta_{1} \lesssim\left(1+\mathrm{R}_{\mathrm{e}}\right)^{-2}, \delta_{2} \lesssim 1$. From (2.31) we get

$$
\begin{aligned}
\mathcal{C}\left(\left(\mathbf{u}_{h}, p_{h}\right),\left(\mathbf{v}_{h}, q_{h}\right)\right) \gtrsim\left\|\left|\mathbf{u}_{h}\right|\right\|^{2}+\left\|\nabla \cdot \mathbf{u}_{h}\right\|_{0, \Omega}^{2}+\left\|\mu^{\frac{1}{2}} \mathbf{u}_{h} \cdot \mathbf{n}\right\|_{+\frac{1}{2}, h, \partial \Omega}^{2}+\left\|\{\mu\}_{w}^{\frac{1}{2}} \llbracket \mathbf{u}_{h} \cdot \mathbf{n} \rrbracket\right\|_{+\frac{1}{2}, h, \Gamma_{\mathrm{int}}}^{2} \\
\quad+\frac{1}{\left(1+\mathrm{R}_{\mathrm{e}}\right)^{2}}\left\|\mu^{-\frac{1}{2}} p_{h}\right\|_{0, \Omega}^{2}+\left\|\mu^{-\frac{1}{2}} \llbracket p_{h} \rrbracket\right\|_{-\frac{1}{2}, h, \mathcal{F}_{h}}^{2} \\
\gtrsim \frac{1}{\left(1+\mathrm{R}_{\mathrm{e}}\right)^{2}}\left\|\left(\mathbf{u}_{h}, p_{h}\right)\right\| \|^{2} .
\end{aligned}
$$

For the second part of the proof, since $\delta_{2} \lesssim 1$, and ||$\left(0, \mu \nabla \cdot \mathbf{u}_{h}\right)||\left|\lesssim\left\|||\left(\mathbf{u}_{h}, p_{h}\right) \mid\right\|\right.$, we have $\left\|\left|\left(\mathbf{v}_{h}, q_{h}\right)\right|\right\| \lesssim$ $\left\|||\left(\mathbf{u}_{h}, p_{h}\right)\left|\left\|+\delta_{1}\right\|\right| \mid\left(\mathbf{v}_{p, h}, 0\right)\right\| \|$. Moreover, using Lemma 2.2 and $\delta_{1} \lesssim\left(1+\mathrm{R}_{\mathrm{e}}\right)^{-2} \lesssim 1$,

$$
\delta_{1}\left|\left\|\left(\mathbf{v}_{p, h}, 0\right)\left|\|\lesssim\| \mu^{\frac{1}{2}} \mathbf{v}_{p, h}\left\|_{1, \Omega} \lesssim\right\| \mu^{-\frac{1}{2}} p_{h}\left\|_{0, \Omega} \lesssim\right\|\right|\left(\mathbf{u}_{h}, p_{h}\right)\right\| \|,\right.
$$

which completes the proof.

Let us comment on this stability result. It states that, at least for small Reynolds numbers, the stability constant with respect to the norm $\left\|\left|\left(\mathbf{u}_{h}, p_{h}\right) \|\right| \mid\right.$ is independent of the coefficients $\nu_{i}, \eta_{i}$, of the problem. Starting from this point, we will show that it is possible to build up suitable preconditioners that are optimal for the problem at hand.

To conclude the present analysis, we briefly summarize the approximation properties of our finite element scheme (we refer to [14] for more details).

Lemma 2.4. Let $(\mathbf{u}, p)$ be the weak solution of (2.5), (2.3), (2.4) with the regularity assumptions (2.19), and let $\left(\mathbf{u}_{h}, p_{h}\right) \in \mathbf{W}_{h}$ be the solution of (2.21). Then, the following a-priori error estimate holds true,

$$
\left\|\left(\mathbf{u}-\mathbf{u}_{h}, p-p_{h}\right)\right\|\left|\lesssim \frac{1}{\left(1+\mathrm{R}_{\mathrm{e}}\right)^{2}} \inf _{\left(\mathbf{z}_{h}, r_{h}\right) \in \mathbf{W}_{h}}\right|\left\|\left(\mathbf{u}-\mathbf{z}_{h}, p-r_{h}\right)\right\| \mid .
$$

In the case of $\mathbb{P}^{1}-\mathbb{P}^{0}$ elements for the velocity and pressure spaces respectively, under the additional regularity assumption $\mathbf{u} \in\left[\cup_{i \in\{l, w\}} H^{2}\left(\Omega_{i}\right)\right]^{d}$ and $p \in \cup_{i \in\{l, w\}} H^{1}\left(\Omega_{i}\right)$ we have,

$$
\|||\left(\mathbf{u}-\mathbf{u}_{h}, p-p_{h}\right)|| \mid \lesssim \frac{h}{\left(1+\mathrm{R}_{\mathrm{e}}\right)^{2}}\left(\left|\mu^{\frac{1}{2}} \mathbf{u}\right|_{\cup \Omega, 2}+\left|\mu^{-\frac{1}{2}} p\right|_{\cup \Omega, 1}\right) .
$$

\section{Solution Strategies For the DiscRete COUPLED Blood Flow PROBlem}

\subsection{An iterative splitting method}

In this section, we present an iterative technique to reduce the solution of the coupled problem (2.21) to a sequence of local subproblems. The main advantages of this approach are the possible parallel implementation, 
and the fact that the iterative splitting strategy can naturally include the Picard's fixed point scheme to treat the nonlinear term.

The proposed iterative method is obtained simply by computing the local solutions $\mathbf{u}_{h, i}, p_{h, i}$ considering in equation (2.21) test functions respectively restricted to the local sub-spaces $\mathbf{V}_{h, i}, Q_{h, i}$. Each of these local subproblems is coupled to the variables on the other sub-domain by interface terms defined on $\Gamma_{\text {int }}$, that are $c^{(b f)}(\cdot, \cdot)$ and $d(\cdot, \cdot)$ defined in (2.11) and (2.12), respectively. Let us introduce the restriction operators $R_{i} \mathbf{v}_{h}=\mathbf{v}_{h, i}$ in $\Omega_{i}$, and $R_{i} \mathbf{v}_{h}=\mathbf{0}$ in $\Omega_{j}$, where $j \in\{l, w\} \backslash i$. Consider the splitting $c^{(b f)}\left(\mathbf{u}_{h}, R_{i} \mathbf{v}_{h}\right)=c_{i i}^{(b f)}\left(\mathbf{u}_{h, i}, \mathbf{v}_{h, i}\right)-$ $c_{i j}^{(b f)}\left(\mathbf{u}_{h, j}, \mathbf{v}_{h, i}\right), d\left(p_{h}, R_{i} \mathbf{v}_{h}\right)=d_{i i}\left(p_{h, i}, \mathbf{v}_{h, i}\right)-d_{i j}\left(p_{h, j}, \mathbf{v}_{h, i}\right), \mathcal{F}^{(b f)}\left(R_{i} \mathbf{v}_{h}\right)=\mathcal{F}_{i}^{(b f)}\left(\mathbf{v}_{h, i}\right)$, where

$$
\begin{aligned}
c_{i i}^{(b f)}\left(\mathbf{u}_{h, i}, \mathbf{v}_{h, i}\right):= & \int_{\Gamma_{\text {int }}}\left(\gamma_{u} h_{F}^{-1}\{\mu\}_{w} \mathbf{u}_{h, i} \cdot \mathbf{n}_{i} \mathbf{v}_{h, i} \cdot \mathbf{n}_{i}\right. \\
& \left.-w_{i} \nu_{i} \mathbf{n}_{i}^{T} \nabla \mathbf{u}_{h, i} \mathbf{n}_{i} \mathbf{v}_{h, i} \cdot \mathbf{n}_{i}-w_{i} \nu_{i} \mathbf{n}_{i}^{T} \nabla \mathbf{v}_{h, i} \mathbf{n}_{i} \mathbf{u}_{h, i} \cdot \mathbf{n}_{i}\right), \\
c_{i j}^{(b f)}\left(\mathbf{u}_{h, j}, \mathbf{v}_{h, i}\right):= & \int_{\Gamma_{\text {int }}}\left(\gamma_{u} h_{F}^{-1}\{\mu\}_{w} \mathbf{u}_{h, j} \cdot \mathbf{n}_{i} \mathbf{v}_{h, i} \cdot \mathbf{n}_{i}\right. \\
& \left.+w_{j} \nu_{j} \mathbf{n}_{i}^{T} \nabla \mathbf{u}_{h, j} \mathbf{n}_{i} \mathbf{v}_{h, i} \cdot \mathbf{n}_{i}-w_{i} \nu_{i} \mathbf{n}_{i}^{T} \nabla \mathbf{v}_{h, i} \mathbf{n}_{i} \mathbf{u}_{h, j} \cdot \mathbf{n}_{i}\right), \\
d_{i i}\left(p_{h, i}, \mathbf{v}_{h, i}\right):= & \int_{\Gamma_{\text {int }}} w_{i} p_{h, i} \mathbf{v}_{h, i} \cdot \mathbf{n}_{i}, \quad d_{i j}\left(p_{h, j}, \mathbf{v}_{h, i}\right):=-\int_{\Gamma_{\text {int }}} w_{j} p_{h, j} \mathbf{v}_{h, i} \cdot \mathbf{n}_{i} .
\end{aligned}
$$

Note that we have $d\left(R_{i} q_{h}, \mathbf{u}_{h}\right)=d_{i i}\left(q_{h, i}, \mathbf{u}_{h, i}\right)+d_{j i}\left(q_{h, i}, \mathbf{u}_{h, j}\right)$. Given the following relaxation operators,

$$
\begin{aligned}
s^{(u)}\left(\mathbf{u}_{h, i}, \mathbf{v}_{h, i}\right) & =\left(\sigma_{u} h_{F}^{-1} \nu_{i} \mathbf{u}_{h, i}, \mathbf{v}_{h, i}\right)_{\Gamma_{\text {int }}}+\left(\sigma_{u} h_{F}^{-1} \mu_{i} \mathbf{u}_{h, i} \cdot \mathbf{n}_{i}, \mathbf{v}_{h, i} \cdot \mathbf{n}_{i}\right)_{\Gamma_{\mathrm{int}}}, \\
s^{(p)}\left(p_{h, i}, q_{h, i}\right) & =\left(\sigma_{p} h_{F}^{-1} \mu_{i}^{-1} p_{h, i}, q_{h, i}\right)_{\Gamma_{\mathrm{int}}},
\end{aligned}
$$

$\sigma_{u}, \sigma_{p}$ being positive relaxation constants, we define the bilinear forms

$$
\begin{aligned}
\mathcal{A}_{i}^{(b f)}\left(\mathbf{u}_{h, i}, \mathbf{v}_{h, i} ; \mathbf{w}_{i}\right) & :=a_{i}^{(b f)}\left(\mathbf{u}_{h, i}, \mathbf{v}_{h, i} ; \mathbf{w}_{i}\right)+c_{i i}^{(b f)}\left(\mathbf{u}_{h, i}, \mathbf{v}_{h, i}\right), \\
\mathcal{B}_{i}\left(p_{h, i}, \mathbf{v}_{h, i}\right) & :=b_{i}\left(p_{h, i}, \mathbf{v}_{h, i}\right)+d_{i i}\left(p_{h, i}, \mathbf{v}_{h, i}\right), \\
\mathcal{C}_{i}\left(\left(\mathbf{u}_{h, i}, p_{h, i}\right),\left(\mathbf{v}_{h, i}, q_{h, i}\right)\right) & :=\mathcal{A}_{i}^{(b f)}\left(\mathbf{u}_{h, i}, \mathbf{v}_{h, i} ; \mathbf{w}_{i}\right)+\mathcal{B}_{i}\left(p_{h, i}, \mathbf{v}_{h, i}\right)-\mathcal{B}_{i}\left(q_{h, i}, \mathbf{u}_{h, i}\right)+j_{i}^{(p)}\left(p_{h, i}, q_{h, i}\right) .
\end{aligned}
$$

Then, the iterative method is defined as follows. For $i \in\{l, w\}, j \in\{l, w\} \backslash\{i\}$, given $\mathbf{u}_{h, i}^{0} \in \mathbf{V}_{h, i}, p_{h, i}^{0} \in Q_{h, i}$, for $m \geq 1$ until convergence we aim to find $\mathbf{u}_{h, i}^{m} \in \mathbf{V}_{h, i}, p_{h, i}^{m} \in Q_{h, i}$ such that

$$
\begin{gathered}
\mathcal{A}_{i}^{(b f)}\left(\mathbf{u}_{h, i}^{m}, \mathbf{v}_{h, i} ; \mathbf{w}_{i}\right)+\mathcal{B}_{i}\left(p_{h, i}^{m}, \mathbf{v}_{h, i}\right)+s^{(u)}\left(\mathbf{u}_{h, i}^{m}, \mathbf{v}_{h, i}\right)= \\
\mathcal{F}_{i}^{(b f)}\left(\mathbf{v}_{h, i}\right)+d_{i j}\left(p_{h, j}^{m-1}, \mathbf{v}_{h, i}\right)+c_{i j}^{(b f)}\left(\mathbf{u}_{h, j}^{m-1}, \mathbf{v}_{h, i}\right) \\
+s^{(u)}\left(\mathbf{u}_{h, i}^{m-1}, \mathbf{v}_{h, i}\right) \quad \forall \mathbf{v}_{h, i} \in \mathbf{V}_{h, i}, \\
\mathcal{B}_{i}\left(q_{h, i}, \mathbf{u}_{h, i}^{m}\right)-s^{(p)}\left(p_{h, i}^{m}, q_{h, i}\right)-j_{i}^{(p)}\left(p_{h, i}^{m}, q_{h, i}\right)=-d_{j i}\left(q_{h, i}, \mathbf{u}_{h, j}^{m-1}\right)-s^{(p)}\left(p_{h, i}^{m-1}, q_{h, i}\right) \quad \forall q_{h, i} \in Q_{h, i} .
\end{gathered}
$$

The convergence of such block-Jacobi iterative scheme, for $\mathbf{w}_{i}=\mathbf{0}$ and for relaxation coefficients $\sigma_{u}, \sigma_{p}$ big enough, has been proved in [14]. A block-Gauss-Seidel scheme can be set up similarly. Moreover, we can modify this scheme letting $\mathbf{w}_{i}=\mathbf{u}_{h, i}^{m-1}$ for $i=l$ in (3.4) obtaining a fixed-point nonlinear iterative method that can be used to account for Navier-Stokes flows. Of course, in this case the convergence result obtained in [14] will not apply; however this strategy proved to be effective in numerical experiments (see Sect. 5).

One interesting feature of the penalty method for interface conditions is to allow an equivalent (symmetric) formulation with respect to the neighboring domains, where the sum of the coupling operators $c^{(b f)}(\cdot, \cdot)$ and $d(\cdot, \cdot)$ defined in equations (2.11), (2.12), contains the numerical interface stress, i.e. the average $\left\{p_{h}-\nu \mathbf{n}^{T} \nu \nabla \mathbf{u}_{h} \mathbf{n}\right\}_{w}$. 
TABLE 1. Number of iterations required to reduce the incremental error $\left\|\left|\left(\mathbf{u}_{h}^{m+1}-\mathbf{u}_{h}^{m}, p_{h}^{m+1}-p_{h}^{m}\right)\right|\right\|$ from $10^{0}$ to $10^{-4}$. Different combinations of the relaxation parameters $\sigma_{u}=\sigma_{p}=\sigma$ and of the weights $w_{w}, w_{l}=1-w_{w}$ are considered.

\begin{tabular}{|c||c|c|c|c|c|c|}
\hline$\sigma$ & $w_{w}=0$ & $w_{w}=0.2$ & $w_{w}=0.4$ & $w_{w}=0.6$ & $w_{w}=0.8$ & $w_{w}=1$ \\
\hline $10^{-1}$ & 1024 & 1013 & 997 & 981 & 963 & 946 \\
$10^{-2}$ & 187 & 132 & 200 & 307 & 363 & 401 \\
$10^{-3}$ & 19 & 160 & 276 & 336 & 425 & 425 \\
0 & 5 & 67 & unstable & unstable & unstable & unstable \\
\hline
\end{tabular}

Using different weights corresponds to biasing the numerical interface stress. In the framework of our splitting method (3.4), this can be interpreted as switching from a Dirichlet-to-Neumann to a Neumann-to-Dirichlet iterative scheme, spanning the whole family of the corresponding convex combinations. In particular, choosing the weights using equation (2.8) in a coupled luminal/transmural blood flow problem, in which typically, $\eta_{w} \gg \nu_{l}$, one finds $w_{w} \simeq 0, w_{l} \simeq 1$. In this case, the splitting method (3.4) is such that the Stokes/Oseen problem receives Dirichlet data for the normal velocity at the interface from the Darcy's problem, and returns the normal component of the normal stresses. In the opposite case, for $w_{w} \simeq 1, w_{l} \simeq 0$, the normal component of the normal stresses are transferred from the Darcy's problem to the Stokes/Oseen's problem, which returns the values of the normal velocity. Obviously, for $\eta_{w} \gg \nu_{l}$ the former scheme is more stable than the latter, since in that case the Darcy's stresses are very sensitive to interface velocity data.

These observations are confirmed by numerical experiments, as described by the following test in dimension two. Let us consider problem $(2.5)$ on $\Omega_{w}=[-1,0]^{2}, \Omega_{l}=[0,1]^{2}$, with constant coefficients $\nu_{l}=1, \eta_{w}=100$. For the sake of simplicity, we consider $\mathbf{w}_{i}=\mathbf{0}$, and assume homogeneous Dirichlet conditions on $[-1,1] \times\{0,1\}$ for the velocity; we prescribe ${ }^{2}$ the normal stresses $p_{w}=1$ on $\{-1\} \times[0,1]$ and consider a mass loss term $\nabla \cdot \mathbf{u}_{l}=-2 x$ instead of $\nabla \cdot \mathbf{u}_{l}=0$. The convergence history of the iterative method (3.4) is quantified by the number of iterations required to satisfy a fixed tolerance on the incremental error, reported in Table 1 for different combinations of weights and relaxation parameters, which in this case were chosen as $\sigma_{u}=\sigma_{p}=\sigma$.

From this experiment, two conclusions about the behavior of our iterative method in the highly heterogeneous case $\eta_{w} \gg \nu_{l}$ can be drawn. First, for large $\sigma$ the convergence rate is slow and dominated by the relaxation operators, regardless of the weights. Second, as $\sigma \rightarrow 0$, the convergence rate improves if $w_{w}$ is sufficiently small. Otherwise, as $\sigma$ gets smaller, deceleration is observed and eventually the iterative method (3.4) may become unstable. This is clearly shown in Table 1, where the unrelaxed scheme with $\sigma=0$ is convergent only for the two smallest $w_{w}$ considered. Notice that the optimal combination is $\sigma=0, w_{w}=0$, and $w_{l}=1$, in which only five iterations are required.

Finally, we point out that the flexibility of choosing the proper weights to optimize the convergence of the iterative method is a remarkable feature of our scheme. This would not be possible with the approach proposed in [7], in which the interface normal stresses have not been treated separately in the normal and tangential component (so that the only admissible weights are $w_{w}=1, w_{l}=0$, i.e. the less performing for our applications), neither with a primal formulation of the Darcy's problem as an elliptic equation for the pressure, see $[18]$.

\subsection{Preconditioning strategy for the solution of the algebraic problem}

Since problem (3.4) has to be solved many times at each time step, we need an efficient solution strategy for the associated algebraic linear system. A very efficient strategy would be to make use of direct methods for the sparse factorization of the linear system, to be precomputed before the iterations are started. However, this approach is very memory consuming and computationally costly; moreover, at each Picard's iteration the factorization step should be updated since the system matrix is modified. A different approach consists

\footnotetext{
${ }^{2}$ This numerical test is based on the exact solution $\mathbf{u}_{w}=\eta_{w}^{-1} \mathbf{e}_{1}, p_{w}=1+\nu_{l}-x-\frac{5}{2}, \mathbf{u}_{l}=\left(\eta_{w}^{-1}-x^{2}\right) \mathbf{e}_{1}, p_{l}=1+(1-2 x) \nu_{l}-\frac{5}{2}$.
} 
in applying a preconditioned Krylov method, such as GMRes, for the iterative solution of the linear systems. In this case, the performance of the method critically depends on the preconditioner. In this section, we will describe some useful preconditioning strategies that can be exploited in this regard. Our considerations will apply for both the local and the global problem. In fact, it is easy to see that the boundedness and stability (inf-sup condition) properties of global problem (2.18) can be proved for the local problems (3.4) as well. For this reason, in this section we will consider an abstract generalized saddle point problem and study the spectral properties of the related algebraic system starting from general boundedness and stability properties. The consequences of such analysis will then apply to both local and global problems.

\subsubsection{An abstract framework for generalized saddle point problems}

Let us denote by $V, Q$ a couple of finite dimensional Hilbert spaces (meant to be generic finite element velocity and pressure spaces, respectively), and let $W=V \times Q$. We consider three abstract bilinear forms $\mathcal{A}: V \times V \rightarrow \mathbb{R}, \mathcal{B}: Q \times V \rightarrow \mathbb{R}$ and $j: Q \times Q \rightarrow \mathbb{R}$. We define

$$
\mathcal{C}((u, p),(v, q))=\mathcal{A}(u, v)+\mathcal{B}(p, v)-\mathcal{B}(q, u)+j(p, q),
$$

and assume the following properties where the product space $W$ is endowed with the norm $\|v, q\|_{W}^{2}=\|v\|_{V}^{2}+$ $\|q\|_{Q}^{2}$.

Hypothesis 3.1. There exist positive numbers $\underline{\alpha}, \bar{\beta}, \underline{\gamma}, \bar{\gamma}$, independent of $u, v, p, q$ such that

$$
\begin{aligned}
\underline{\alpha}\|v\|_{V}^{2} & \leq \mathcal{A}(v, v), \\
\bar{\beta}\|p\|_{Q}\|v\|_{V} & \geq \mathcal{B}(p, v), \\
\underline{\gamma}\|u, p\|_{W} & \leq \sup _{v, q \neq 0} \frac{\mathcal{C}((u, p),(v, q))}{\|v, q\|_{W}}, \\
\bar{\gamma}\|u, p\|_{W} & \geq \sup _{v, q \neq 0} \frac{\mathcal{C}((u, q),(v, q))}{\|v, q\|_{W}} .
\end{aligned}
$$

Analogously, there exists $\bar{\gamma}^{\prime} \leq \bar{\gamma}$ such that

$$
\bar{\gamma}^{\prime}\|u, p\|_{W} \geq \sup _{q \neq 0} \frac{\mathcal{B}(u, q)-j(p, q)}{\|q\|_{Q}} .
$$

Remark 3.1. The existence of $\bar{\gamma}^{\prime}$ trivially follows from (3.9) with $\bar{\gamma}^{\prime}=\bar{\gamma}$. However, we are considering the case in which a better estimate of $\bar{\gamma}^{\prime}$ (i.e., smaller than $\bar{\gamma}$ ) may be available.

In this section, with little abuse of notation, we will use the same symbols to denote $u \in V, p \in Q$, and the vectors $u \in \mathbb{R}^{\operatorname{dim}(V)}, p \in \mathbb{R}^{\operatorname{dim}(Q)}$ of the components with respect to the bases of the spaces $V, Q$. According to this notation, we focus on the following abstract linear system,

$$
C\left[\begin{array}{l}
u \\
p
\end{array}\right]=\left[\begin{array}{cc}
A & B^{T} \\
-B & J
\end{array}\right]\left[\begin{array}{l}
u \\
p
\end{array}\right]=\left[\begin{array}{l}
f_{u} \\
f_{p}
\end{array}\right],
$$

whose blocks are related to the bilinear forms as follows,

$$
\mathcal{A}(u, v)=(v, A u), \quad \mathcal{B}(q, u)=(q, B u), \quad j(p, q)=(q, J p),
$$

where $(\cdot, \cdot)$ is the Euclidean scalar product.

First, let us show that problem (2.21) fits the abstract framework introduced so far. 
Lemma 3.2. The bilinear forms $\mathcal{A}^{(b f)}(\cdot, \cdot ; \cdot), \mathcal{B}(\cdot, \cdot)$ and $\mathcal{C}(\cdot, \cdot)$ defined in (2.15), (2.16), (2.20) respectively, satisfy Hypothesis 3.1 with $V=\mathbf{V}_{h}, Q=Q_{h}$ and the following norms,

$$
\begin{aligned}
\left\|\mathbf{v}_{h}\right\|_{V}^{2} & =\left|\left\|\mathbf{v}_{h}\right\|\right|^{2}+\left\|\{\mu\}_{w}^{\frac{1}{2}} \llbracket \mathbf{v}_{h} \rrbracket \cdot \mathbf{n}_{\Gamma}\right\|_{+\frac{1}{2}, h, \Gamma_{\mathrm{int}}}^{2}+\sum_{i=l, w}\left[\left\|\mu_{i}^{\frac{1}{2}} \nabla \cdot \mathbf{v}_{h, i}\right\|_{0, \Omega_{i}}^{2}+\left\|\mu_{i}^{\frac{1}{2}} \mathbf{v}_{h, i} \cdot \mathbf{n}\right\|_{+\frac{1}{2}, h, \Gamma_{i}^{D}}^{2}\right] \\
\left\|q_{h}\right\|_{Q}^{2} & =\sum_{i=l, w}\left[\left\|\mu_{i}^{-\frac{1}{2}} q_{h, i}\right\|_{0, \Omega_{i}}^{2}+\left\|\mu_{i}^{-\frac{1}{2}} \llbracket q_{h, i} \rrbracket\right\|_{-\frac{1}{2}, h, \mathcal{F}_{h, i}}^{2}\right]
\end{aligned}
$$

with constants $\bar{\beta}=C, \bar{\gamma}=C\left(1+\mathrm{R}_{\mathrm{e}}\right), \underline{\gamma}=C\left(1+\mathrm{R}_{\mathrm{e}}\right)^{-2}, \bar{\gamma}^{\prime}=C$ and $\underline{\alpha}=C \max \left\{h^{2}, \min _{i} \frac{\nu_{i}}{\mu_{i}}\right\}$, where $C$ denotes a generic constant independent on $h, \nu_{i}, \mu_{i}$.

Proof. Owing to Lemma 2.1 (boundedness), observing that the convective term appears in $\mathcal{A}^{(b f)}$ only, we have that inequalities (3.7) and (3.10) are verified with constants $\bar{\beta} \lesssim 1$ and $\bar{\gamma}^{\prime} \lesssim 1$, whilst (3.9) is satisfied with $\bar{\gamma} \lesssim\left(1+R_{e}\right)$. Lemma 2.3 (stability) ensures that (3.8) holds with $\underline{\gamma} \gtrsim\left(1+R_{e}\right)^{-2}$. Concerning inequality (3.6), recall the positivity result of Lemma 2.3. Therefore, it will suffice to find a positive constant $K$ such that

$$
\sum_{i=l, w}\left(\left\|\eta_{i}^{\frac{1}{2}} \mathbf{v}_{h, i}\right\|_{0, \Omega_{i}}^{2}+\left\|\nu_{i}^{\frac{1}{2}} \nabla \mathbf{v}_{h, i}\right\|_{0, \Omega_{i}}^{2}\right) \gtrsim K \sum_{i=l, w}\left\|\mu_{i}^{\frac{1}{2}} \nabla \cdot \mathbf{v}_{h, i}\right\|_{0, \Omega_{i}}^{2}
$$

To this purpose, we have to distinguish between the case $\min _{i} \nu_{i}>0$ and $\min _{i} \nu_{i}=0$. In the former case, (3.12) holds true with $K=\min _{i} \frac{\nu_{i}}{\mu_{i}}$. In the latter case, thanks to the inverse inequalities (2.24), (3.12) is satisfied with $K=h^{2}$.

The local problems (3.4) enjoy the same boundedness, positivity and stability estimates as the global problem with respect to the local norms. Hence, Lemma 3.2 holds also locally, as stated below.

Lemma 3.3. The bilinear forms $\mathcal{A}_{i}^{(b f)}(\cdot, \cdot ; \cdot), \mathcal{B}_{i}(\cdot, \cdot)$ and $\mathcal{C}_{i}(\cdot, \cdot)$ defined in (3.1), (3.2), (3.3) respectively, satisfy Hypothesis 3.1 with $V=\mathbf{V}_{h, i}, Q=Q_{h, i}$ and the following norms,

$$
\begin{aligned}
\left\|\mathbf{v}_{h, i}\right\|_{V}^{2}= & \left\|\eta_{i}^{\frac{1}{2}} \mathbf{v}_{h, i}\right\|_{0, \Omega_{i}}^{2}+\left\|\nu_{i}^{\frac{1}{2}} \nabla \mathbf{v}_{h, i}\right\|_{0, \Omega_{i}}^{2}+\left\|\mu_{i}^{\frac{1}{2}} \nabla \cdot \mathbf{v}_{h, i}\right\|_{0, \Omega_{i}}^{2} \\
& +\left\|\nu_{i}^{\frac{1}{2}} \mathbf{v}_{h, i}\right\|_{+\frac{1}{2}, h, \Gamma_{i}^{D}}^{2}+\left\|\nu_{i}^{\frac{1}{2}} \mathbf{n} \times \mathbf{v}_{h, i}\right\|_{+\frac{1}{2}, h, \Gamma_{\mathrm{int}}}^{2} \\
& +\left\|\mu_{i}^{\frac{1}{2}} \mathbf{v}_{h, i} \cdot \mathbf{n}\right\|_{+\frac{1}{2}, h, \Gamma_{i}^{D}}^{2}+\left\|\left(\mu_{i} w_{i}\right)^{\frac{1}{2}} \mathbf{v}_{h, i} \cdot \mathbf{n}\right\|_{+\frac{1}{2}, h, \Gamma_{\mathrm{int}}}^{2} \\
\left\|q_{h, i}\right\|_{Q}^{2}= & \left\|\mu_{i}^{-\frac{1}{2}} q_{h, i}\right\|_{0, \Omega_{i}}^{2}+\left\|\mu_{i}^{-\frac{1}{2}} \llbracket q_{h, i} \rrbracket\right\|_{-\frac{1}{2}, h, \mathcal{F}_{h, i}}^{2},
\end{aligned}
$$

with constants $\bar{\beta}=C, \bar{\gamma}=C\left(1+\mathrm{R}_{\mathrm{e}}\right), \underline{\gamma}=C\left(1+\mathrm{R}_{\mathrm{e}}\right)^{-2}, \bar{\gamma}^{\prime}=C$ and $\underline{\alpha}=C \max \left\{h^{2}, \frac{\nu_{i}}{\mu_{i}}\right\}$, where $C$ denotes a generic constant independent on $h, \nu_{i}, \mu_{i}$.

The eigenvalue analysis of the generalized saddle point system (3.11) has been carried out in several works; we cite among others $[23,37,43]$, for symmetric $A$, and $[24,39]$ for the nonsymmetric case. The concept behind almost all such eigenvalue analysis is norm-equivalence (or, more generally, field-of-values equivalence, see [24,39]), which implies spectral equivalence.

We provide here an original and alternative approach that exploits singular value analysis to prove the norm and spectral equivalence in the non-symmetric case. We start directly from the stability and boundedness properties of the global form $\mathcal{C}$. The SVD tools that we will use are recalled in the following lemma. 
Lemma 3.4. Let $M \in \mathbb{R}^{N \times N}$ be a square matrix. Let $\sigma_{1}(M) \geq \sigma_{2}(M) \geq \ldots \geq \sigma_{N}(M) \geq 0$ be the singular values and $\lambda_{n}(M), n=1, \ldots, N$, the eigenvalues of $M$. Then,

$$
\begin{array}{ll}
\sigma_{N}(M)=\inf _{y \neq 0} \sup _{x \neq 0} \frac{(y, M x)}{\|y\|_{2}\|x\|_{2}} ; & \sigma_{1}(M)=\sup _{y \neq 0} \sup _{x \neq 0} \frac{(y, M x)}{\|y\|_{2}\|x\|_{2}}, \\
\sigma_{N}(M) \leq\left|\lambda_{n}(M)\right| \leq \sigma_{1}(M), & n=1, \ldots, N .
\end{array}
$$

Proof. For inequalities (3.13) see for instance [22], Theorem 7.3.10. Estimates (3.14) are standard as well and directly follow from (3.13) taking $y=e_{n}$ a unit left eigenvector of $M$ associated with $\lambda_{n}(M)=\lambda_{n}\left(M^{T}\right)$.

\subsubsection{Preconditioners for the Schur complement.}

Typically, one may consider block-diagonal or block-triangular preconditioners of system (3.11), as discussed in [19]. We shall focus on a special instance of block-triangular (left) preconditioners. In particular, we will consider the preconditioner $P$ based on the exact $A$ block and a suitable approximation $\hat{S}$ of the Schur complement $S:=B A^{-1} B^{T}+J$,

$$
P^{-1}=\left[\begin{array}{cc}
A & 0 \\
B & -\hat{S}
\end{array}\right]^{-1}=\left[\begin{array}{cc}
A^{-1} & 0 \\
\hat{S}^{-1} B A^{-1} & -\hat{S}^{-1}
\end{array}\right]
$$

such that the preconditioned system matrix reads,

$$
P^{-1} C=\left[\begin{array}{cc}
I & A^{-1} B^{T} \\
0 & \hat{S}^{-1} S
\end{array}\right]
$$

Of course, we could replace a suitable approximation $\hat{A}$ of $A$ in the expression of the preconditioner; in the sequel, we restrict our analysis to exact solution of the momentum equation. From (3.16), we have that the eigenvalues of the preconditioned system are

$$
\lambda_{n}\left(P^{-1} C\right)= \begin{cases}1 & n=1, \ldots, \operatorname{dim} V \\ \lambda_{n}\left(\hat{S}^{-1} S\right) & n=\operatorname{dim}(V)+1, \ldots, \operatorname{dim}(W) .\end{cases}
$$

In the symmetric case ( $A^{T}=A$, which implies $S^{T}=S$ and $S$ positive), the convergence of Krylov iterative methods for symmetric systems, such as PCG or MinRes, depends on the ratio between the largest and the smallest eigenvalue of the preconditioned matrix $\hat{S}^{-1} S$. In particular, if this ratio does not depend on $\operatorname{dim}(W)$, the preconditioner $\hat{S}$ will be optimal (i.e. the number of iterations of the Krylov method will be independent on the mesh size). Optimality of $\hat{S}$ for methods for unsymmetric/undefinite systems, such as GMRes, requires field-of-values (FOV) equivalence between $\hat{S}$ and $S$. However, experience shows rapid convergence of GMRes if the eigenvalues of the preconditioned system matrix are clustered in a bounded region away from zero. From these observations, it is clear that finding a preconditioner $\hat{S}$ with good spectral properties is a crucial step in order to efficiently solve system (3.11).

The goal of this section is to show that the Schur complement is spectrally equivalent to a suitable preconditioner. The simplest case of spectrally equivalent preconditioner is given by the (Grammian) symmetric positive definite matrix $H_{Q}$ inducing the scalar product in $Q$, satisfying $\left(p, H_{Q} p\right)=\|p\|_{Q}^{2}$. For instance, in [11] estimates of the eigenvalues of the Schur complement are obtained starting from boundedness, positivity and stabilized inf-sup conditions for a similar generalized saddle point problem. Using a separate analysis of the symmetric and skew-symmetric part of the Schur complement, the authors show that $\hat{S}=H_{Q}$ is an optimal preconditioner for $S$. Here we provide a similar result by means of SVD analysis. 
Theorem 3.5. Under Assumption 3.1, the eigenvalues of $H_{Q}^{-1} S$ are localized as follows,

$$
\lambda_{n}\left(H_{Q}^{-1} S\right) \in\left\{z \in \mathbb{C}: \underline{\gamma} \leq|z| \leq \bar{\gamma}^{\prime} \sqrt{1+\left(\frac{\bar{\beta}}{\underline{\alpha}}\right)^{2}}\right\} .
$$

Proof. For each $p \in Q$, let $u_{p} \in V$ be defined by

$$
\mathcal{A}\left(u_{p}, v\right)+\mathcal{B}(p, v)=0 \quad \forall v \in V, \quad \text { that is, } \quad u_{p}=-A^{-1} B^{T} p .
$$

Taking $u=u_{p}$ in $(3.5)$ makes $\mathcal{C}\left(\left(u_{p}, p\right),(v, q)\right)=j^{(p)}(p, q)-\mathcal{B}\left(u_{p}, q\right)=(q, S p)$ independent of $v$; hence, using $(3.8)$ and (3.10),

$$
\underline{\gamma}\left\|u_{p}, p\right\|_{W} \leq \sup _{(v, q) \neq 0} \frac{(q, S p)}{\|v, q\|_{W}}=\sup _{q \neq 0} \frac{(q, S p)}{\|q\|_{Q}} \leq \bar{\gamma}^{\prime}\left\|u_{p}, p\right\|_{W} .
$$

From (3.17) and (3.6), (3.7) we have,

$$
\underline{\alpha}\left\|u_{p}\right\|_{V}^{2} \leq \mathcal{A}\left(u_{p}, u_{p}\right)=-\mathcal{B}\left(p, u_{p}\right) \leq \bar{\beta}\|p\|_{Q}\left\|u_{p}\right\|_{V},
$$

so that $\left\|u_{p}\right\|_{V} \leq \frac{\bar{\beta}}{\underline{\alpha}}\|p\|_{Q}$, yielding the following estimate,

$$
\|p\|_{Q} \leq\left\|u_{p}, p\right\|_{W} \leq \sqrt{1+\left(\frac{\bar{\beta}}{\underline{\alpha}}\right)^{2}}\|p\|_{Q}
$$

and equation (3.18) becomes

$$
\underline{\gamma}\|p\|_{Q} \leq \sup _{q \neq 0} \frac{(q, S p)}{\|q\|_{Q}} \leq \bar{\gamma}^{\prime} \sqrt{1+\left(\frac{\bar{\beta}}{\underline{\alpha}}\right)^{2}}\|p\|_{Q} .
$$

Now, let $\tilde{p}=H_{Q}^{\frac{1}{2}} p, \tilde{q}=H_{Q}^{\frac{1}{2}} q$; using $\|p\|_{Q}=\|\tilde{p}\|_{2},\|q\|_{Q}=\|\tilde{q}\|_{2}$ and (3.20), we have

$$
\underline{\gamma} \leq \sup _{\tilde{q} \neq 0} \frac{(\tilde{q}, \tilde{S} \tilde{p})}{\|\tilde{q}\|_{2}\|\tilde{p}\|_{2}} \leq \bar{\gamma}^{\prime} \sqrt{1+\left(\frac{\bar{\beta}}{\underline{\alpha}}\right)^{2}} \quad \forall \tilde{p} \in Q
$$

where $\tilde{S}=H_{Q}^{-\frac{1}{2}} S H_{Q}^{-\frac{1}{2}}$.

Thanks to Lemma 3.4, applying (3.14), (3.13) with $M=\tilde{S}$, using estimates (3.21) and the fact that $\lambda_{n}(\tilde{S})=$ $\lambda_{n}\left(H_{Q}^{-1} S\right)$, the eigenvalue bounds follow.

Notice that matrix $H_{Q}$ is well-conditioned, as stated below.

Lemma 3.6. Matrix $H_{Q}$ is spectrally equivalent to the mass matrix $M_{Q}$ defined by $\left(p, M_{Q} q\right)=\left(\mu^{-\frac{1}{2}} p, \mu^{-\frac{1}{2}} q\right)_{L^{2}}$, uniformly with respect to $h, \eta, \nu$.

Proof. Thanks to the inverse inequality (2.24), the norms $\|q\|_{Q}$ and $\left\|\mu^{-\frac{1}{2}} q\right\|_{L^{2}}$ are equivalent, uniformly with respect to $h, \eta, \nu$.

Combining Lemma 3.3 with Theorem 3.5, we obtain the following result, establishing the optimality of preconditioner $(3.15)$ with $\hat{S}=H_{Q}$ or $\hat{S}=M_{Q}$. 
Corollary 3.7. Let $C$ be the matrix associated to the bilinear form $\mathcal{C}$ of (3.5), $P$ be the preconditioner given by (3.15) with $\hat{S}=H_{Q}$ or $\hat{S}=M_{Q}$, and assume that Hypothesis 3.1 holds. Then, we have

$$
\frac{\max \left|\lambda_{n}\left(P^{-1} C\right)\right|}{\min \left|\lambda_{n}\left(P^{-1} C\right)\right|}=\frac{\max \left|\lambda_{n}\left(\hat{S}^{-1} S\right)\right|}{\min \left|\lambda_{n}\left(\hat{S}^{-1} S\right)\right|} \lesssim\left(1+\mathrm{R}_{\mathrm{e}}\right)^{2}\left(\max \left\{\min _{i} \frac{\nu_{i}}{\mu_{i}}, h^{2}\right\}\right)^{-1} .
$$

Again, spectral equivalence does not imply FOV equivalence. FOV equivalence could be obtained in specific cases [28], however our goal was not to focus on a specific (GMRes) iterative method to solve the Schur complement problem. In general, based on the eigenvalue analysis, we can expect that $\hat{S}=H_{Q}\left(\hat{S}=M_{Q}\right)$ will be a good preconditioner in the viscous case only. In fact, the preconditioned Krylov solver will be efficient at least for moderate $\mathrm{R}_{\mathrm{e}}$ and in the low $\mu / \nu$ regime. For instance, if the resistive term $\eta$ dominates the viscosity $\nu$, then the spectral ratio (3.22) becomes proportional to $h^{-2}$. To overcome such difficulties, we introduce below a modified preconditioner that is specifically tailored to the inviscid terms (Darcy's) in the problem.

Let us treat separately the viscous and resistive terms by introducing the preconditioners $\hat{S}_{\nu}, \hat{S}_{\eta}$ defined as follows:

$$
\hat{S}_{\nu}=M_{Q_{\frac{1}{\nu}}}, \quad \hat{S}_{\eta}=B \hat{M}_{V_{\eta}}^{-1} B^{T}+J,
$$

where $M_{Q_{\frac{1}{\nu}}}$ is defined by $\left(p, M_{Q_{\frac{1}{\nu}}} q\right)=\left(\nu^{-\frac{1}{2}} p, \nu^{-\frac{1}{2}} q\right)_{L^{2}}, \hat{M}_{V_{\eta}}$ is the diagonal matrix obtained by summing row-wise (lumping) the elements of the matrix $M_{V_{\eta}}$ such that $\left(u, M_{V_{\eta}} v\right)=\left(\eta^{\frac{1}{2}} u, \eta^{\frac{1}{2}} v\right)_{L^{2}}$.

As for Lemma 3.6, it is easy to see that $\hat{S}_{\nu}$ is equivalent to the preconditioner $H_{Q}$. Conversely, $\hat{S}_{\eta}$ is an approximation of the Schur complement of a pure Darcy's problem. We propose to construct $\hat{S}_{\eta}$ taking the approximation of the exact Schur complement $S=B M_{V_{\eta}}^{-1} B^{T}+J$ by lumping the matrix $M_{V_{\eta}}$. Since the lumped matrix is diagonal, it is easily inverted, so that the construction of $\hat{S}_{\eta}$ is straightforward and based on immediately available matrices.

The two preconditioners can be combined, yielding the following compound preconditioner,

$$
\hat{S}_{\nu, \eta}^{-1}:=\hat{S}_{\nu}^{-1}+\hat{S}_{\eta}^{-1} .
$$

In the classical setting of the Stokes' equation, it is well known that this preconditioner is optimal [10]. However, as we will see in the next section, $\hat{S}_{\eta}$ is not easily dealt with by classical ILU/IC solvers, which can limit its applicability as Schur complement preconditioner.

\subsubsection{Monolithic preconditioners}

Instead of addressing the usual Schur complement, we now look at (3.11) as a monolithic system and we propose a convenient preconditioner. To this purpose, we introduce the Grammian matrix of the space $W$, satisfying $\left((v, q), H_{W}(v, q)\right)=\|v, q\|_{W}^{2}$. This is a symmetric, positive definite, block-diagonal matrix, whose blocks are the Grammian matrices $H_{V}$ and $H_{Q}$ associated to spaces $V$ and $Q$ respectively. Thanks to our boundedness and stability estimates, which are uniform not only in $h$ but in $\nu, \eta$ as well, a suitable monolithic preconditioner for matrix $C$ is given by $H_{W}$, as stated by the following result.

Theorem 3.8 (monolithic preconditioner spectral equivalence). Under Assumptions 3.1, the eigenvalues of $H_{W}^{-1} C$ satisfy

$$
\lambda_{n}\left(H_{W}^{-1} C\right) \in\{z \in \mathbb{C}: \underline{\gamma} \leq|z| \leq \bar{\gamma}\} .
$$

Proof. We proceed as in the case of Theorem 3.5 and the result immediately follows for matrix $\tilde{C}=H_{W}^{-\frac{1}{2}} C H_{W}^{-\frac{1}{2}}$ from the combination of (3.8) and (3.9) with Lemma 3.4. The proof follows since $\tilde{C}$ and $H_{W}^{-1} C$ are similar.

Owing to Lemmas 3.2 and 3.3, it is straightforward to conclude that $H_{W}$ is an optimal preconditioner for the single domain problems (3.4) as well as for the coupled problem (2.21). The choice of the monolithic preconditioner $H_{W}$ has several advantages. First, the preconditioned system turns out to be insensitive of 
TABLE 2. Number of GMRes iteration required to reduce the error by a factor $10^{-9}$. Three cases are considered: (a) $\nu=1, \eta=0, \mathrm{R}_{\mathrm{e}}=0$; (b) $\nu=1, \eta=1, \mathrm{R}_{\mathrm{e}}=0$; (c) $\nu=1, \eta=1$, $\mathrm{R}_{\mathrm{e}}=1$.

\begin{tabular}{|c||c|c||c|c||c|c|}
\hline \multicolumn{1}{|c||}{} & \multicolumn{2}{c||}{ (a) } & \multicolumn{2}{c||}{ (b) } & \multicolumn{2}{c|}{$(\mathrm{c})$} \\
\hline$h$ & $\hat{S}_{\nu}$ & $\hat{S}_{\nu, \eta}$ & $\hat{S}_{\nu}$ & $\hat{S}_{\nu, \eta}$ & $\hat{S}_{\nu}$ & $\hat{S}_{\nu, \eta}$ \\
\hline $1 / 20$ & 18 & - & 18 & 25 & 18 & 25 \\
$1 / 40$ & 21 & - & 21 & 28 & 21 & 28 \\
$1 / 80$ & 22 & - & 22 & 29 & 22 & 29 \\
$1 / 160$ & 22 & - & 23 & 29 & 23 & 29 \\
\hline
\end{tabular}

TABLE 3. Number of GMRes iteration required to reduce the error by a factor $10^{-9}$, and memory coefficient $C_{\text {MEM. }}$. (a) $\nu=8.5 \times 10^{-5}, \eta=0, \mathrm{R}_{\mathrm{e}}=0$; (b) $\nu=8.5 \times 10^{-5}, \eta=10^{3}$, $\mathrm{R}_{\mathrm{e}}=0 ;(\mathrm{c}) \nu=8.5 \times 10^{-5}, \eta=10^{3}, \mathrm{R}_{\mathrm{e}}=300$.

\begin{tabular}{|c||c|c||c|c||c|c||c|}
\hline \multicolumn{1}{|l||}{} & \multicolumn{2}{c||}{ (a) } & \multicolumn{2}{c||}{ (b) } & \multicolumn{2}{c||}{$(\mathrm{c})$} & \\
\hline$h$ & $\hat{S}_{\nu}$ & $\hat{S}_{\nu, \eta}$ & $\hat{S}_{\nu}$ & $\hat{S}_{\nu, \eta}$ & $\hat{S}_{\nu}$ & $\hat{S}_{\nu, \eta}$ & $C_{\mathrm{MEM}}$ \\
\hline $1 / 10$ & 14 & - & 55 & 19 & 58 & 45 & 1.00 \\
$1 / 20$ & 18 & - & 105 & 25 & 147 & 89 & 1.19 \\
$1 / 40$ & 21 & - & 243 & 34 & 403 & 167 & 1.64 \\
\hline
\end{tabular}

the mesh characteristic size and of the heterogeneity of the coefficients $\nu$ and $\eta$, as long as inequalities (3.8) and (3.9) in Hypothesis 3.1 are satisfied. They actually are, at least if Neumann boundary conditions are prescribed on a non-empty subset of the external boundaries of each subdomain, which is the fundamental assumption of Theorem 2.2. Second, the monolithic preconditioner does not require to resort to any (possibly inexact) second order pressure problem. We are instead obliged to do so, by introducing matrix $\hat{S}_{\eta}$, at least when viscous terms are dominated by mass terms, if the Schur complement approach is adopted.

\subsection{Numerical experiments}

\subsubsection{Performance of the Schur complement preconditioners}

Let us check the performance of the preconditioners $\hat{S}_{\nu}$ and $\hat{S}_{\nu, \eta}$ introduced in Section 3.2. To this end, we consider 2D model problems on one domain only, using lowest order $(k=1)$ polynomials. In particular, we consider problem $(2.5)$ on $\Omega=[0,1]^{2}$, with constant coefficients $\nu>0, \eta \geq 0$. As boundary conditions, we assign a parabolic velocity profile $\mathbf{U}=4 y(1-y)$ on the inflow $\Gamma_{\text {in }}(x=0)$, no-slip conditions at the walls $y=0$, $y=1$, and prescribe the stress $\sigma \mathbf{n}=\mathbf{0}$ at the outflow $\Gamma_{\text {out }}(x=1)$. We set $\mathbf{w}=\nu \mathrm{R}_{\mathrm{e}} y(1-y) \mathbf{e}_{x}$, where $\mathrm{R}_{\mathrm{e}}$ is the Reynolds number.

Let us consider the number of GMRes iterations required to solve this one-domain problem for several values of the parameters $\eta, \nu$ and $\mathbf{w}$, and for the two proposed preconditioners. In the numerical experiments reported in Table 2 we address problems that are dominated by viscosity, in which $\eta \leq \nu$ and $\mathrm{R}_{\mathrm{e}} \leq 1$. In this region, the spectral ratio estimate (3.22) is mesh and parameter independent. As expected, the preconditioner $\hat{S}_{\nu}$ is optimal regardless of the mesh size and parameter values in the selected range. The same consideration holds for $\hat{S}_{\nu, \eta}$ (in this case the number of iterations is slightly bigger).

In the experiments reported in Table 3 we consider more realistic data, using values in typical ranges of hemodynamics. Specifically, we consider low viscosity and medium Reynolds number, $\eta=10^{3} \gg \nu=8.5 \times$ $10^{-5}$ and $R_{e}=300$. These values are obtained after suitable rescaling from the physiological data reported in Section 5. According to (3.22), since $\left(\max \left\{\min _{i} \nu_{i} / \mu_{i}, h^{2}\right\}\right)^{-1}=h^{-2}$ (for not too small values of $h$ ), the spectral ratio estimate is inversely dependent on the mesh characteristic size. As expected, the GMRes iterations required with $\hat{S}_{\nu}$, which scale as $\sqrt{h^{-2}}$, almost double at each mesh refinement by bisection. Conversely, 
TABLE 4. The smallest and the largest magnitude eigenvalues $\left[\lambda_{\min }, \lambda_{\max }\right]$ of the generalized eigenvalue problem $C w=\lambda H_{W} w$, for the coupled heterogeneous Darcy-Stokes' problem of Section 3.3.2, and the number of $H_{W}$-preconditioned GMRes iterations required to reduce the error by a factor $10^{-10}$ for the linear system $C w=f$, for different values of $h$ and $C_{\mu}=\mu_{w} / \mu_{l}$.

\begin{tabular}{|c||c|c|c||c|c|c||c|c|c|}
\hline \multicolumn{1}{|c||}{$h$} & \multicolumn{3}{c||}{$C_{\mu}=10^{0}$} & \multicolumn{3}{c||}{$C_{\mu}=10^{-3}$} & \multicolumn{3}{c|}{$C_{\mu}=10^{-6}$} \\
\hline & $\lambda_{\min }$ & $\lambda_{\max }$ & It. & $\lambda_{\min }$ & $\lambda_{\max }$ & It. & $\lambda_{\min }$ & $\lambda_{\max }$ & It. \\
\hline $1 / 8$ & 0.178 & 2.023 & 156 & 0.178 & 2.000 & 134 & 0.178 & 2.000 & 146 \\
\hline $1 / 16$ & 0.178 & 1.996 & 175 & 0.178 & 1.987 & 145 & 0.178 & 1.987 & 184 \\
\hline $1 / 32$ & 0.177 & 1.995 & 177 & 0.177 & 1.995 & 147 & 0.177 & 1.995 & 176 \\
\hline
\end{tabular}

preconditioning by $\hat{S}_{\nu, \eta}$ significantly improves the performance of GMRes, especially at low Reynolds numbers. A practical drawback of using the latter preconditioner is that it requires solving linear systems associated to the matrix $\hat{S}_{\eta}$, which suffers from fill-in effect. To quantify the extra memory requirements, the indicator $C_{\mathrm{MEM}}$, defined as the ratio between the memory allocated for an Incomplete Cholesky (IC) factorization of $\hat{S}_{\eta}$ and the memory occupied by $\hat{S}_{\eta}$,

$$
C_{\mathrm{MEM}}=\frac{\text { Memory }\left[I C\left(\hat{S}_{\eta}\right)\right]}{\operatorname{Memory}\left[\hat{S}_{\eta}\right]}
$$

is reported in Table 3. The Incomplete Cholesky matrix is computed by choosing the fill-in threshold such that the number of iterations to solve the preconditioned system associated to $\hat{S}_{\eta}$ is constant (15 iterations). The coefficient $C_{\mathrm{MEM}}$ is not constant: it grows as the square root of the system size, which means that memory allocation is superlinear.

\subsubsection{Performance of the monolithic preconditioner}

In this section we are going to show that the proposed method is robust with respect to the coefficients $\eta_{i}$ and $\nu_{i}$, and that the monolithic preconditioner is optimal (for low Reynolds numbers). Let us consider a (bidomain) problem analogous to that of Section 3.1, using lowest order $(k=1)$ polynomials, where we assign different values to the viscosity coefficient $\nu$. Precisely, let $\Omega_{w}=[-1,0] \times[0,1], \Omega_{l}=[0,1] \times[0,1], \nu_{w}=0$, $\eta_{w}=1, \nu_{l}=\nu>0, \eta_{l}=0, \mathbf{w}_{i}=\mathbf{0}, i=w, l$. $\left(\right.$ i.e. $\left.\mathrm{R}_{\mathrm{e}}=0\right), p_{w}=\nu-\frac{1}{2}$ on $[0,1] \times\{1,0\}, p_{l}=-\frac{3}{2}-\nu$ on $\{1\} \times[0,1]$, $\mathbf{u}_{w} \cdot \mathbf{n}_{w}=0$ on $[-1,0] \times\{1,0\}$ and $\mathbf{u}_{l}=\mathbf{0}$ on $[0,1] \times\{1,0\}$. As interface conditions on $\Gamma_{\text {int }}=\{0\} \times[0,1]$, we apply (2.4). We point out that in this simple case we have $\Gamma_{s, i}=\Gamma_{\text {out }, l}=\emptyset$. The stabilization parameters are set to $\gamma_{u}=\gamma_{p}=2$.

We aim to verify the spectral equivalence of matrix $C$ associated to the global bilinear form $\mathcal{C}$ of problem (2.21) with the monolithic preconditioner $H_{W}$ (Thm. 3.8), for different values of the mesh size $h$ and of the viscosity $\nu$, and discuss the convergence rate of the preconditioned GMRes method. To this end, we compute (through the eigs command provided by Matlab 7.5.0, i.e. using arpack) the smallest and largest magnitude eigenvalues $\lambda_{\min }, \lambda_{\max }$ of the generalized eigenvalue problem $C w=\lambda H_{W} w$. Moreover, we report the number of preconditioned GMRes iterations required to compute the approximate solution within the tolerance of $10^{-10}$ on the preconditioned residual.

As expected, the results presented in Table 4 for various combinations of the heterogeneity ratio $C_{\mu}:=$ $\mu_{l} / \mu_{w}=\nu / 1$ and the mesh characteristic size $h$, show that $H_{W}$ is an optimal preconditioner for matrix $C$. Indeed, $\lambda_{\min }$ and $\lambda_{\max }$ are independent of $C_{\mu}$, and the number of GMRes iterations is always less than 190 for all considered cases. This also confirms the robustness of the stability estimate of Theorem 2.3 with respect to coefficients. As a consequence, $H_{W}$ can be successfully applied as a preconditioner to solve the global problem by Krylov iterative solvers such as GMRes. The computational cost of each preconditioning step is even reduced with respect to the Schur complement approach, since $H_{W}$ is efficiently dealt with (symmetric positive definite and block-diagonal). However, the number of GMRes iterations is generally increased, at least in the viscosity dominated regime. Again, the splitting method (3.4) and/or the preconditioning techniques based on the Schur complement introduced in Section 3.2 may be useful to further reduce the cost of each iteration. 


\section{Modelling AND APPROXimation of MASS TRANSFER}

We assume that the drug released by the stent behaves as a passive scalar. By consequence, our drug release model features just one chemical species, the drug, whose concentration is governed by standard advectiondiffusion equations. Precisely, the governing equations for drug concentration in the lumen and in the arterial wall, denoted with $c_{l}(t, \mathbf{x})$ and $c_{w}(t, \mathbf{x})$ respectively, read as follows,

$$
\frac{\partial c_{i}}{\partial t}+\nabla \cdot\left(-D_{i} \nabla c_{i}+\mathbf{u}_{i} c_{i}\right)=0 \text { in } \Omega_{i}, \text { with } i=l, w
$$

where $D_{i}$ is a diffusion coefficient and $\mathbf{u}_{i}$ is the advection field (the blood/plasma velocity). Equations (4.1) are complemented by an initial condition prescribing the initial state of the concentration in the blood stream and the arterial walls, i.e. $c_{i}(t=0)=0$ in $\Omega_{i}$, and suitable boundary conditions. For the arterial lumen, $\Omega_{l}$, on the inflow boundary $\Gamma_{\text {in }}$ we prescribe $c_{l}=0$ since the blood does not contain drug proximally to the stent. Assuming that the outflow boundary is far enough from the stent, we can neglect any diffusive effects across this section and set $\nabla c_{l} \cdot \mathbf{n}_{l}=0$ on $\Gamma_{\text {out. }}$. Also for the arterial wall we prescribe $\nabla c_{w} \cdot \mathbf{n}_{w}=0$ on $\Gamma_{\text {ext }} \cup \Gamma_{\text {cut }}$.

The coupling between equations (4.1) is provided by the following conditions, expressing the continuity of the concentration and of the total normal flux (we refer to [25] for a discussion of the applicability of these standard conditions to the specific biomedical problem at hand),

$$
\left\{\begin{aligned}
-D_{l} \nabla c_{l} \cdot \mathbf{n}+\mathbf{u}_{l} \cdot \mathbf{n} c_{l}=-D_{w} \nabla c_{w} \cdot \mathbf{n}+\mathbf{u}_{w} \cdot \mathbf{n} c_{w} & \text { on } \Gamma_{\text {int }} \\
c_{l}=c_{w}, & \text { on } \Gamma_{\text {int }} .
\end{aligned}\right.
$$

Finally, particular attention should be payed to the condition on the interface with the stent, because it is primarily responsible in determining the drug release rate. Precisely, the latter is defined as the (purely diffusive) mass flux at the interface $\Gamma_{s, i}$, i.e. $J=-D_{i} \nabla c_{i} \cdot \mathbf{n}_{i}$ (recall that $\mathbf{u} \cdot \mathbf{n}=0$ at the interface with the stent). We remind that DES for cardiovascular applications are miniaturized metal structures that are coated with a micro-film containing the drug that will be locally released into the arterial walls for healing purposes. The thickness of this film generally lays within the range of microns. Owing to the fact that the stent coating is extremely thin, we apply the model proposed in [42] where the following formula for the release rate has been derived,

$$
J(t, \mathbf{x})=\varphi(t)\left(c_{s}^{0}-c_{i}\right) \text { on } \Gamma_{s, i} \quad \text { with } i=l, w,
$$

being $c_{s}^{0}$ the initial drug charge of the stent that is equal to the unity in the nondimensional setting for the concentration. Given the thickness of the stent coating, $\Delta l$, and its diffusion parameter, $D_{s}$, the scaling function $\varphi(t)$ is defined as follows,

$$
\varphi(t)=\frac{2 D_{s}}{\Delta l} \sum_{n=0}^{\infty} \mathrm{e}^{-(n+1 / 2)^{2} k t} \quad \text { with } k=\pi^{2} D_{s} / \Delta l^{2} .
$$

Owing to (4.3), the boundary condition on $\Gamma_{s, l}$ and $\Gamma_{s, w}$ for equation (4.1) turns out to be the following Robin type condition,

$$
-D_{i} \nabla c_{i} \cdot \mathbf{n}_{i}+\varphi(t)\left(c_{s}^{0}-c_{i}\right)=0 \text { on } \Gamma_{s, i} \quad \text { with } i=l, w
$$

We notice that the validity of the coupled mass transfer model (4.1), (4.2), (4.4) is subject to some simplifications concerning the complexity of the structure and mass transport characteristics of the arterial wall.

First, we assume that the drug does not react with the arterial walls. Hence, we are neglecting the chemical phenomena that involve the drug as a ligand and suitable sites of the extracellular matrix as receptors. It is well known that such phenomena may strongly influence the distribution of the drug into the arterial walls, as discussed in [35,41]. However, it is not definitely clarified how to translate these phenomena into equations and how to feed them with parameters. Furthermore, under the aforementioned assumption that the arterial wall can be described as a homogeneous and isotropic medium, the drug diffusivity parameter is considered to be uniform and it will be taken from [25]. As regards the transmission conditions between $\Omega_{l}$ and $\Omega_{w}$ we notice that we have neglected the presence of the endothelium, a single layer of specific cells at the interface 
between the lumen and the arterial wall. The presence of such layer can be addressed by means of a Robin type boundary condition accounting for the characteristic endothelial resistance to mass transfer. Indeed, the endothelium represents a selective barrier for the absorption of molecules, such as lipids, into the arterial wall. However, clinical evidence shows that the endothelium is severely injured after an angioplastic procedure with stent grafting. Then, our simplistic assumption, which neglects the endothelial resistance, is basically motivated by the lack of information about the corresponding residual functionality of this layer of cells.

\subsection{Finite element approximation}

For the discretization of problem (4.1), (4.2), (4.4) we apply the numerical scheme introduced in [8], taking the discrete blood and plasma filtration velocities as advection fields. The only difference is that in our case the global velocity field may have a jump across the interface $\Gamma_{\text {int }}$. In this regard, we adopt a simple modification of the scheme presented in [8] by considering the average of the advection fields at the interface.

Let us define the following trial and test spaces,

$$
V_{h, i}:=\left\{v_{h}: v_{h} \in C\left(\Omega_{i}\right):\left.v_{h}\right|_{K} \in \mathbb{P}_{k}(K), \forall K \in \mathcal{T}_{h, i}\right\}, \quad V_{h}:=\bigoplus_{i=1}^{N} V_{h, i}
$$

and introduce the following bilinear forms,

$$
\begin{aligned}
a_{i}^{(m t)}\left(c_{h, i}, d_{h, i}\right):= & \int_{\Omega_{i}}\left[\left(\frac{1}{\tau}-\nabla \cdot \mathbf{u}_{h, i}\right) c_{h, i} d_{h, i}+D_{i} \nabla c_{h, i} \cdot \nabla d_{h, i}-c_{h, i} \mathbf{u}_{h, i} \cdot \nabla d_{h, i}\right] \\
& +\int_{\mathcal{F}_{h, i}} \gamma_{\mathbf{u}} h_{F}^{2}\left\|\mathbf{u}_{h, i} \cdot \mathbf{n}\right\|_{L^{\infty}(E)} \llbracket \nabla c_{h, i} \cdot \mathbf{n} \rrbracket \llbracket \nabla d_{h, i} \cdot \mathbf{n} \rrbracket \\
& +\int_{\partial \Omega_{i} \cap \Gamma_{\text {in }}}\left(\frac{1}{2}\left(\left|\mathbf{u}_{h, i} \cdot \mathbf{n}_{i}\right|-\mathbf{u}_{h, i} \cdot \mathbf{n}_{i}\right)+\gamma_{c} D_{i} h_{F}^{-1}\right) c_{h, i} d_{h, i} \\
& +\int_{\partial \Omega_{i} \cap \Gamma_{\text {in }}}\left(-D_{i} \nabla c_{h, i} \cdot \mathbf{n}_{i} d_{h, i}-D_{i} \nabla d_{h, i} \cdot \mathbf{n}_{i} c_{h, i}\right) \\
& +\int_{\partial \Omega_{i} \backslash \Gamma_{\mathrm{int}}} \mathbf{u}_{h, i} \cdot \mathbf{n}_{i} c_{h, i} d_{h, i}+\int_{\Gamma_{s, i}} \varphi\left(t^{n}\right) c_{h, i} d_{h, i}, \quad i=l, w, \\
c^{(m t)}\left(c_{h}, d_{h}\right):=\int_{\Gamma_{\mathrm{int}}} & \left(\frac{1}{2}\left(\left|\mathbf{u}_{h} \cdot \mathbf{n}\right|-\left\{\mathbf{u}_{h} \cdot \mathbf{n}\right\}\right)+\gamma_{c}\{D\}_{w} h_{F}^{-1}\right) \llbracket c_{h} \rrbracket \llbracket d_{h} \rrbracket \\
& +\int_{\Gamma_{\mathrm{int}}}\left(\left\{\mathbf{u}_{h} \cdot \mathbf{n}\right\}\left\{c_{h}\right\}_{w} \llbracket d_{h} \rrbracket-\left\{D \nabla c_{h} \cdot \mathbf{n}\right\}_{w} \llbracket d_{h} \rrbracket-\left\{D \nabla d_{h} \cdot \mathbf{n}\right\}_{w} \llbracket c_{h} \rrbracket\right),
\end{aligned}
$$

where $\gamma_{c}>0, \gamma_{\mathbf{u}}>0$ are stabilization coefficients and the weights $w_{i}$ in $\{D\}_{w}$ and $\left\{D \nabla c_{h} \cdot \mathbf{n}\right\}_{w}$ are now chosen as $w_{i}=\frac{D_{j}}{D_{i}+D_{j}}, i \in\{l, w\}, j \neq i$, as discussed in [8]. Again, we omit the explicit notation for the time level $n$. Then, the mass transfer problem for drug release consists in finding at each time step a couple of functions $\left(c_{h, l}, c_{h, w}\right)=c_{h} \in V_{h}$ such that

$$
\mathcal{A}^{(m t)}\left(c_{h}, d_{h}\right)=\mathcal{F}^{(m t)}\left(d_{h}\right), \quad \forall d_{h} \in V_{h},
$$

where

$$
\begin{aligned}
\mathcal{A}^{(m t)}\left(c_{h}, d_{h}\right) & :=\sum_{i=l, w} a_{i}^{(m t)}\left(c_{h, i}, d_{h, i}\right)+c^{(m t)}\left(c_{h}, d_{h}\right), \\
\mathcal{F}^{(m t)}\left(d_{h}\right) & :=\sum_{i=l, w}\left[\frac{1}{\tau} \int_{\Omega_{i}} c_{h, i}^{(n-1)} d_{h, i}+\int_{\Gamma_{s, i}} \varphi\left(t^{n}\right) c_{s}^{0} d_{h, i}\right] .
\end{aligned}
$$




\section{Application to VASCUlar HEMODYNAMiCS AND BIOCHEMICAL TRANSPORT}

In this section, we will present the numerical simulation of blood flow and mass transport in a realistic threedimensional vascular district containing a stent. Specifically, the geometry of lumen $\Omega_{l}$ and of the wall $\Omega_{w}$ are obtained by the simulation of the mechanical expansion of a stent similar to the coronary Cordis BX-Velocity (Johnson \& Johnson, Interventional System, Warren, NJ, USA), as described in [44]. The radius of the lumen is about $1.55 \mathrm{~mm}$, the thickness of the wall is $0.5 \mathrm{~mm}$.

The finite element methods described in the previous sections were implemented in a $\mathrm{C}++$ code using the lowest order finite element pair $(k=1)$.

According to standard physiological data [40], the dynamic viscosity of blood is set to $\nu_{l}=3 \mathrm{~mm}^{2} \cdot \mathrm{s}^{-1} \mathrm{and}$ the inverse permeability of the arterial wall to $\eta_{w}=10^{12} \mathrm{~s}^{-1}$. Moreover, at the inflow of the artery we impose a parabolic velocity profile (max velocity $270 \mathrm{~mm} \cdot \mathrm{s}^{-1}$, corresponding to a physiological mean flow rate for the considered artery). The remaining model parameters are $\nu_{w}=0, \eta_{l}=0, \mathbf{f}_{i}=\mathbf{0}(i \in\{l, w\})$.

\subsection{Analysis of blood flow}

We performed a blood flow simulation, in the steady case, using the iterative method (3.4) in the block-GaussSeidel form. The stabilization and relaxation parameters were chosen as follows: $\gamma_{u}=\gamma_{p}=\gamma_{\text {int }}=20, \sigma_{u}=0$, $\sigma_{p}=10^{-8}$. We recall that, in general, convergence of the iterative method is ensured only if all stabilization parameters are large enough. On the other hand, we have already observed that smaller relaxation parameters $\sigma_{u}, \sigma_{p}$ are allowed thanks to the tilted choice of the weights (2.8) and lead to faster convergence of the iterative method. Moreover, we applied in (3.4) the nonlinear update $\mathbf{w}_{l}=\mathbf{u}_{l}^{m-1}$ to solve the full Navier-Stokes problem. Convergence was observed in 15 Picard's iterations where the Darcy's equation is solved first, before solving the linearized Navier-Stokes problem. Iterations were stopped when the triple norm of the increment was less than $10^{-8}$. The preconditioner (3.24) was employed at each nonlinear iteration. PCG was used to solve the (symmetric positive definite) Schur complement of the Darcy's problem in the wall; the iteration count was always less than 9. GMRes was used for the (unsymmetric) Oseen problem in the lumen; the related iteration count was ranging from 590 (first Picard's iteration) to less than 10.

As discussed in [13,44], we observe (Fig. 2) that the luminal flow downstream the stent struts is fully threedimensional and shows recirculations, vortexes and secondary motions. Obviously, it is very important to capture these features for the subsequent simulation of the drug release process. In Figure 3, the blood pressure in each subdomain and the filtration velocity in the arterial wall are represented. Notice how the method is able to capture the main features of the coupled problem, characterized by fast flow and small pressure variations in the lumen, slow flow and high pressure gap in the wall, and differences in blood velocity of about five orders of magnitude.

\subsection{Analysis of drug release}

We simulate the release of heparin from a stent coated with a substrate that opposes only moderate resistance to drug release. According to the experimental investigations presented in [29], this corresponds to $D_{l}=1.5 \times 10^{-4} \mathrm{~mm}^{2} \cdot \mathrm{s}^{-1}, D_{w}=7.7 \times 10^{-6} \mathrm{~mm}^{2} \cdot \mathrm{s}^{-1}$, and a large diffusivity of the drug into the stent coating, which is set to $D_{s}=10^{-8} \mathrm{~mm}^{2} \cdot \mathrm{s}^{-1}$.

The numerical simulation, based on the scheme proposed in Section 4, shows that the drug released into the lumen is very quickly lost in the blood stream. Indeed, the peak of drug concentration into the lumen is reached about $40 \mathrm{~s}$ after the beginning of the process. Conversely, the drug dynamics into the arterial walls is much slower. Again, the proposed computational method is able to capture the main features of the coupled problem. In Figure 4, a concentration isosurface shows how the drug is distributed in the blood stream near the device. The luminal concentration rapidly vanishes moving off the stent. Notice that blood flow downstream the stent causes a certain amount of drug to keep close the wall and eventually enter it, as already discussed in [13]. The major contribution is of course given by the diffusive flux through the interface $\Gamma_{s, w}$ between the device and the wall. However, the advantage of our method is that it is able to compute the combined effects 

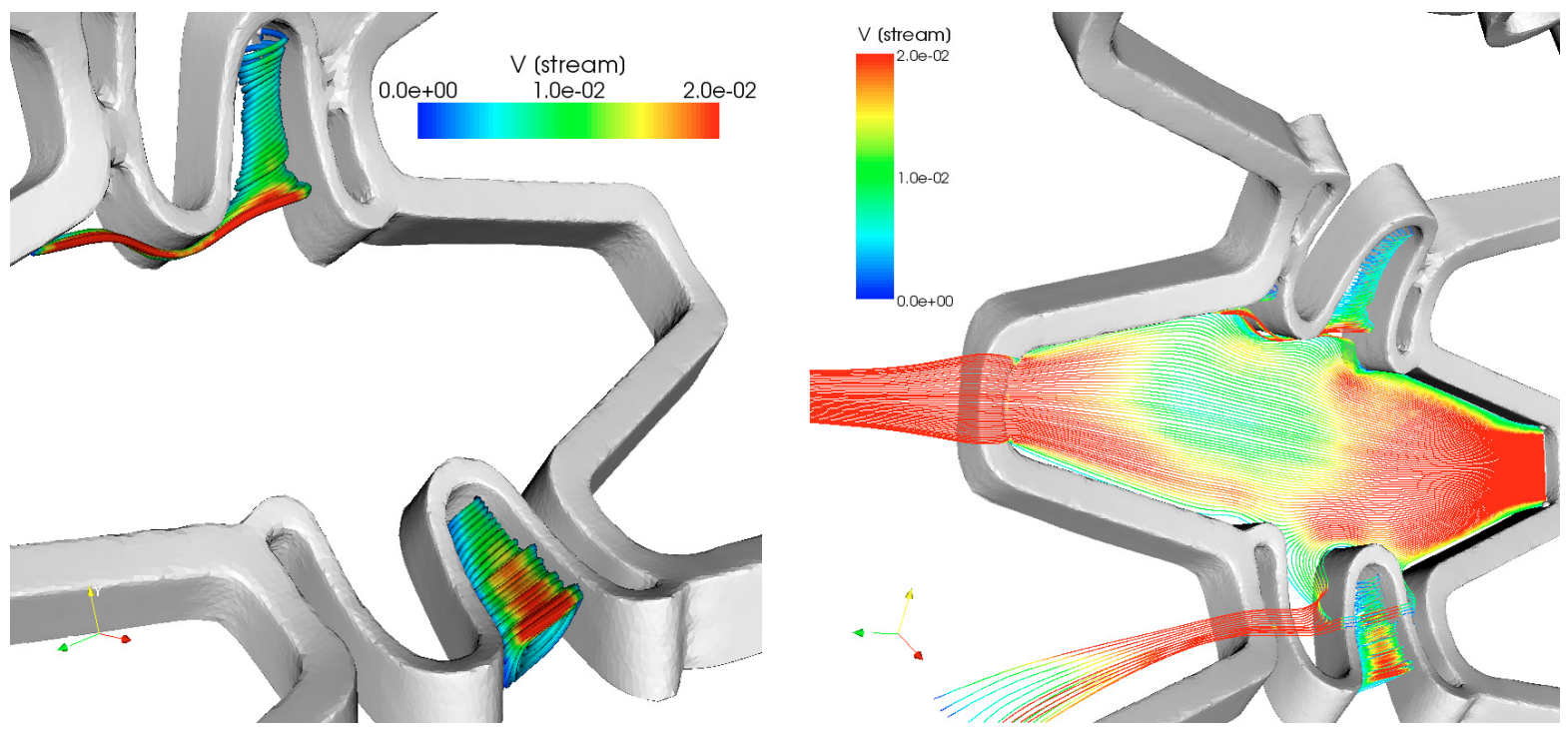

FIGURE 2. The interaction between the stent and the blood flow visualized by means of streamlines. The proximal section is located on the right while the distal section is on the left. Velocity units correspond to $200 \mathrm{~mm} \cdot \mathrm{s}^{-1}$.
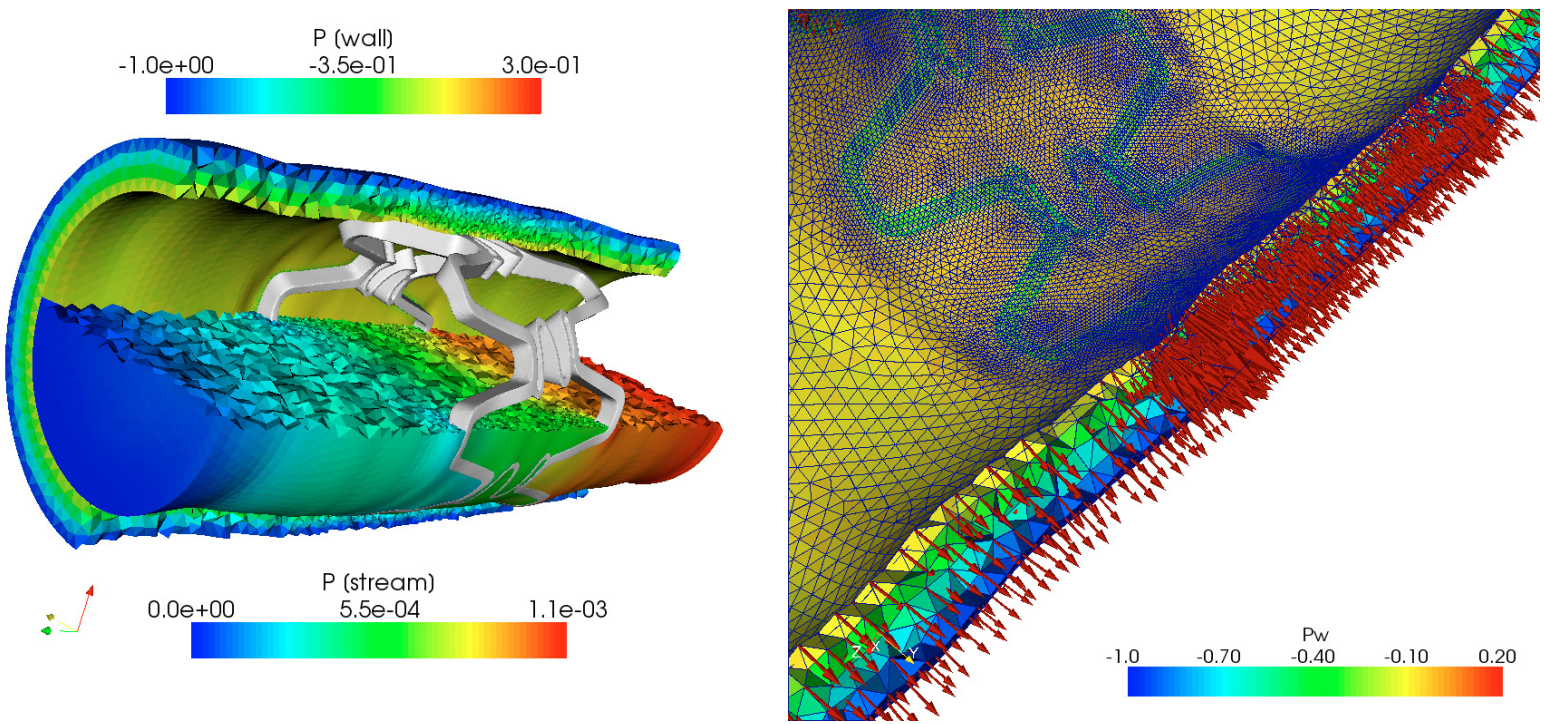

Figure 3. On the left: pressure distribution in the domains $\Omega_{i}$ (pressure units correspond to $70 \mathrm{~mm} \mathrm{Hg}$ ). On the right: filtration velocity (velocity units correspond to $200 \mathrm{~mm} \cdot \mathrm{s}^{-1}$ ) and intramural pressure.

of luminal/wall blood flow on the release pattern, which has not been considered before. Hence, in order to evaluate the impact of the filtration velocity in the wall, we performed two simulations of the release process, first considering only diffusion in $\Omega_{w}$ and imposing no-slip interface conditions on $\Gamma_{\text {int }}$, then performing a fully coupled simulation of blood flow and plasma filtration. The results are reported in Figure 5 . We observe that in the very first hours, considering the transmural filtration results in increased drug delivery to the wall. 


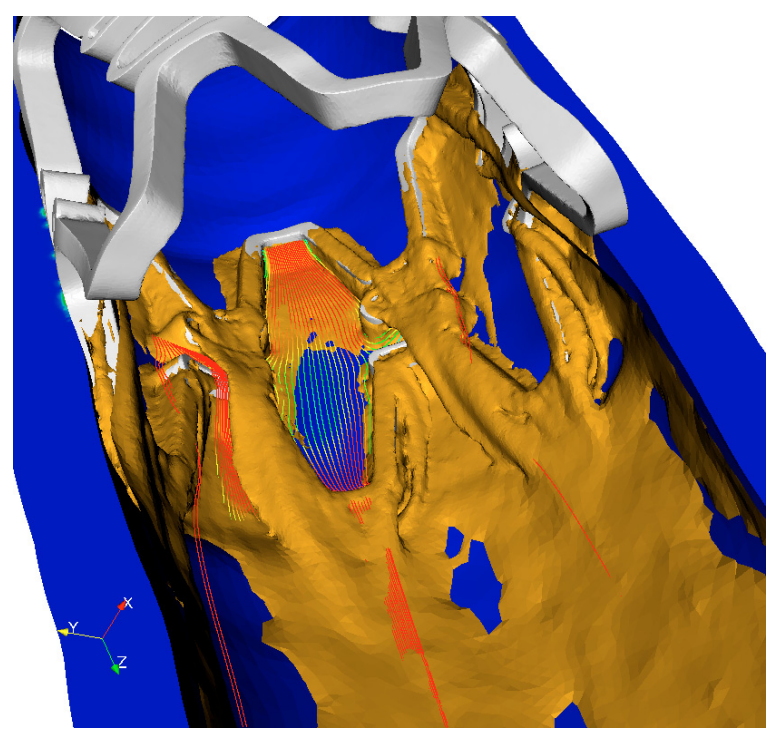

Figure 4 . The iso-surface corresponding to the value $10^{-5} c_{s}^{0}$ for the drug concentration in the arterial lumen and contour plots into the arterial walls, after $40 \mathrm{~s}$ from the beginning of the process. Streamlines of luminal velocity emphasize the role of fluid-dynamics in influencing the distribution of the drug in the blood near the walls.

This is due the enhanced drug penetration associated to the advective term. Such effect reduces the local drug accumulation in the neighborhood of the stent, facilitating further drug release into the artery. As previously mentioned, the first source for drug absorption is the interface between the artery and the stent, but also the drug trapped in the recirculation zones and in the near-wall regions of the lumen may interact with the wall. The presence of intramural plasma filtration may slightly increase the role of this secondary absorption pathway. However, after a few hours, the drug reaches the external boundary $\Gamma_{\text {ext }}$ and the process is reversed, since the outgoing plasma is carrying out a certain amount of drug. Hence, in the long term the filtration velocity causes a diminished drug deposition into the walls.

\section{Conclusions}

We have considered a robust numerical method for the approximation of heterogeneous incompressible flow problems. We have studied its fundamental properties, such as stability, relating them to the coefficients of the problem, in order to emphasize the robustness of the method. We have presented an iterative method to split the coupled heterogeneous problem in possibly homogeneous local problems, showing that the flexible choice of the weights allows for considerable speed-up of the iterations. Moreover, we have proposed and studied suitable preconditioners for the solution of the global and local problems. In particular, the spectral properties of the proposed preconditioners have been investigated, and their performances have been assessed by means of numerical experiments.

Finally, the proposed method has been applied for the computation of fully coupled blood flow in a stented coronary artery, considering the Navier-Stokes equations in the arterial lumen and the Darcy's filtration law in the arterial wall. The computed blood velocities in both domains have been used to perform drug release simulations and study the drug deposition into the walls. The method proved to be robust with respect to the difference of about five orders of magnitude in luminal/filtration velocities, capturing the complex features of the luminal flow around the stent. Moreover, it has been found that transmural filtration has a significant 


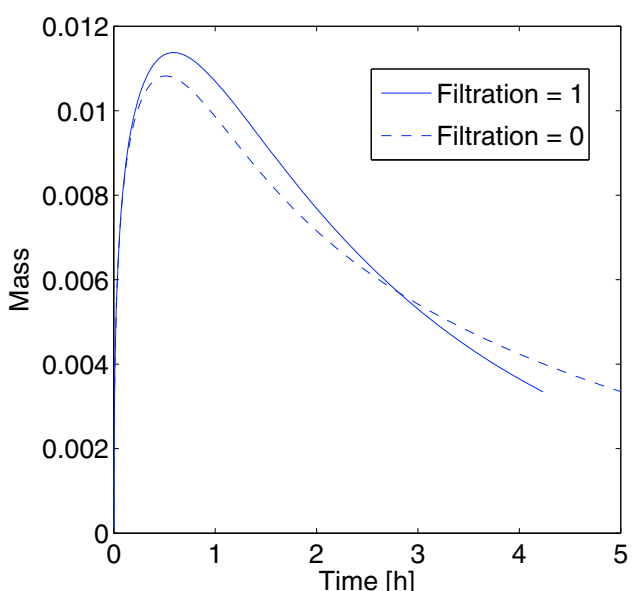

(a)

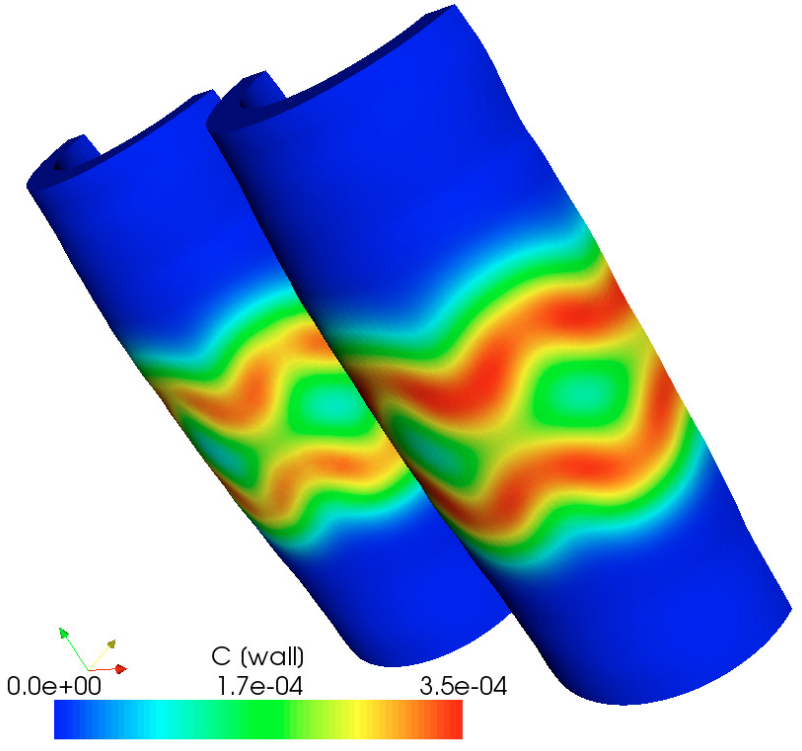

(b)

Figure 5. (a) Time course of the total amount of drug in the arterial wall in two cases, considering only diffusion (dashed line) in the mass transport equations, and including the filtration velocity $\mathbf{u}_{w}$ (solid line). (b) Comparison of drug concentration with (left) and without (right) transmural filtration velocity after $4 \mathrm{~h}$ from the beginning of the process.

impact on drug delivery. Numerical simulations suggest that plasma filtration improves the release process only in the early stages, and promotes the migration of the drug outside the arterial tissue in the long term.

\section{REFERENCES}

[1] S. Badia and R. Codina, Unified stabilized finite element formulations for the Stokes and the Darcy problems. SIAM J. Numer. Anal. 47 (2009) 1971-2000.

[2] B. Balakrishnan, A.R. Tzafriri, P. Seifert, A. Groothuis, C. Rogers and E.R. Edelman, Strut position, blood flow, and drug deposition. Implications for single and overlapping drug-eluting stents. Circulation 111 (2005) 2958-2965.

[3] R. Balossino, F. Gervaso, F. Migliavacca and G. Dubini, Effects of different stent designs on local hemodynamics in stented arteries. J. Biom. 41 (2008) 1053-1061.

[4] J.M. Boland and R.A. Nicolaides, Stability of finite elements under divergence constraints. SIAM J. Numer. Anal. 20 (1983) $722-731$.

[5] J.H. Bramble, J.E. Pasciak and O. Steinbach, On the stability of the $L^{2}$ projection in $H^{1}(\Omega)$. Math. Comp. $71(2002) 147-156$.

[6] E. Burman, Pressure projection stabilizations for Galerkin approximations of Stokes' and Darcy's problem. Numer. Meth. Partial Diff. Equ. 24 (2008) 127-143.

[7] E. Burman and P. Hansbo, A unified stabilized method for Stokes' and Darcy's equations. J. Comput. Appl. Math. 198 (2007) $35-51$.

[8] E. Burman and P. Zunino, A domain decomposition method based on weighted interior penalties for advection-diffusionreaction problems. SIAM J. Numer. Anal. 44 (2006) 1612-1638.

[9] E. Burman, M.A. Fernández and P. Hansbo, Continuous interior penalty finite element method for Oseen's equations. SIAM J. Numer. Anal. 44 (2006) 1248-1274.

[10] J. Cahouet and J.-P. Chabard, Some fast 3D finite element solvers for the generalized Stokes problem. Int. J. Numer. Methods Fluids 8 (1988) 869-895.

[11] C. Calgaro, P. Deuring and D. Jennequin, A preconditioner for generalized saddle point problems: application to 3D stationary Navier-Stokes equations. Numer. Methods Partial Diff. Equ. 22 (2006) 1289-1313. 
[12] C. D'Angelo and A. Quarteroni, On the coupling of 1D and 3D diffusion-reaction equations. Application to tissue perfusion problems. Math. Models Methods Appl. Sci. 18 (2008) 1481-1504.

[13] C. D'Angelo and P. Zunino, A numerical study of the interaction of blood flow and drug release from cardiovascular stents, in Numerical Mathematics and Advanced Applications - Proceedings of ENUMATH 2007, Springer, Berlin (2008) 75-82.

[14] C. D'Angelo and P. Zunino, A finite element method based on weighted interior penalties for heterogeneous incompressible flows. SIAM J. Numer. Anal. 47 (2009) 3990-4020.

[15] C. D'Angelo and P. Zunino, Multiscale models of drug delivery by thin implantable devices, in Applied and industrial mathematics in Italy III, Ser. Adv. Math. Appl. Sci. 82, World Sci. Publ. (2009).

[16] C. D'Angelo and P. Zunino, Numerical approximation with Nitsche's coupling of transient Stokes'/Darcy's flow problems applied to hemodynamics. Technical report, MOX, Department of Mathematics, Politecnico di Milano (submitted).

[17] M.C. Delfour, A. Garon and V. Longo, Modeling and design of coated stents to optimize the effect of the dose. SIAM J. Appl. Math. 65 (2005) 858-881.

[18] M. Discacciati, A. Quarteroni and A. Valli, Robin-Robin domain decomposition methods for the Stokes Darcy coupling. SIAM J. Numer. Anal. 45 (2007) 1246-1268.

[19] H.C. Elman, D.J. Silvester and A.J. Wathen, Finite elements and fast iterative solvers: with applications in incompressible fluid dynamics. Numerical Mathematics and Scientific Computation, Oxford University Press, New York (2005).

[20] L. Formaggia, A. Quarteroni and A. Veneziani Eds., Cardiovascular mathematics - Modeling and simulation of the circulatory system, MSEA Modeling, Simulation and Applications 1. Springer-Verlag Italia, Milan (2009).

[21] L. Formaggia, S. Minisini and P. Zunino, Modeling erosion controlled drug release and transport phenomena in the arterial tissue. Math. Models Methods Appl. Sci. (to appear).

[22] R.A. Horn and C.R. Johnson, Matrix analysis. Cambridge University Press, Cambridge (1990), Corrected reprint of the 1985 original.

[23] A. Klawonn, Block-triangular preconditioners for saddle point problems with a penalty term. SIAM J. Sci. Comput. 19 (1998) 172-184. Special issue on iterative methods (Copper Mountain, CO, 1996).

[24] A. Klawonn and G. Starke, Block triangular preconditioners for nonsymmetric saddle point problems: field-of-values analysis. Numer. Math. 81 (1999) 577-594.

[25] V.B. Kolachalama, A.R. Tzafriri, D.Y. Arifin and E.R. Edelman, Luminal flow patterns dictate arterial drug deposition in stent-based delivery. J. Control Release 133 (2009) 24-30.

[26] W.J. Layton, F. Schieweck and I. Yotov, Coupling fluid flow with porous media flow. SIAM J. Numer. Anal. 40 (2003) $2195-2218$.

[27] S.L. Lee and C.W. Gear, Second-order accurate projective integrators for multiscale problems. J. Comput. Appl. Math. 201 (2007) $258-274$.

[28] D. Loghin and A.J. Wathen, Analysis of preconditioners for saddle-point problems. SIAM J. Sci. Comput. 25 (2004) $2029-2049$.

[29] M.A. Lovich and E.R. Edelman, Mechanisms of transmural heparin transport in the rat abdominal aorta after local vascular delivery. Circ. Res. 77 (1995) 1143-1150.

[30] F. Migliavacca, L. Petrini, M. Colombo, F. Auricchio and R. Pietrabissa, Mechanical behavior of coronary stents investigated through the finite element method. J. Biomech. 35 (2002) 803-811.

[31] L. Petrini, F. Migliavacca, F. Auricchio and G. Dubini, Numerical investigation of the intravascular coronary stent flexibility. J. Biomech. 37 (2004) 495-501.

[32] G. Pontrelli and F. de Monte, Mass diffusion through two-layer porous media: an application to the drug-eluting stent. Int. J. Heat Mass Transfer 50 (2007) 3658-3669.

[33] K.R. Rajagopal, On a hierarchy of approximate models for flows of incompressible fluids through porous solids. Math. Models Methods Appl. Sci. 17 (2007) 215-252.

[34] P. Saffman, On the boundary condition at the surface of a porous media. Stud. Appl. Math. 50 (1971) 292-315.

[35] D.V. Sakharov, L.V. Kalachev and D.C. Rijken, Numerical simulation of local pharmacokinetics of a drug after intravascular delivery with an eluting stent. J. Drug Targ. 10 (2002) 507-513.

[36] Ch. Schwab, $p$ - and hp-Finite Element Methods - Theory and applications in solid and fluid mechanics. Numerical Mathematics and Scientific Computation, Oxford University Press, New York (1998).

[37] D. Silvester and A. Wathen, Fast iterative solution of stabilised Stokes systems. II. Using general block preconditioners. SIAM J. Numer. Anal. 31 (1994) 1352-1367.

[38] J.S. Soares and P. Zunino, A mixture model for water uptake, degradation, erosion and drug release from polydisperse polymeric networks. Biomaterials 31 (2010) 3032-3042.

[39] G. Starke, Field-of-values analysis of preconditioned iterative methods for nonsymmetric elliptic problems. Numer. Math. 78 (1997) $103-117$.

[40] S. Tada and J.M. Tarbell, Internal elastic lamina affects the distribution of macromolecules in the arterial wall: a computational study. Am. J. Physiol. Heart Circ. Physiol. 287 (2004) H905-H913.

[41] A.R. Tzafriri and E.R. Edelman, On the validity of the quasi-steady state approximation of bimolecular reactions in solution. J. Theor. Biol. 233 (2005) 343-350. 
[42] C. Vergara and P. Zunino, Multiscale boundary conditions for drug release from cardiovascular stents. Multiscale Model. Simul. 7 (2008) 565-588.

[43] A. Wathen and D. Silvester, Fast iterative solution of stabilised Stokes systems. I. Using simple diagonal preconditioners. SIAM J. Numer. Anal. 30 (1993) 630-649.

[44] P. Zunino, C. D'Angelo, L. Petrini, C. Vergara, C. Capelli and F. Migliavacca, Numerical simulation of drug eluting coronary stents: Mechanics, fluid dynamics and drug release. Comput. Methods Appl. Mech. Eng. 198 (2009) 3633-3644. 